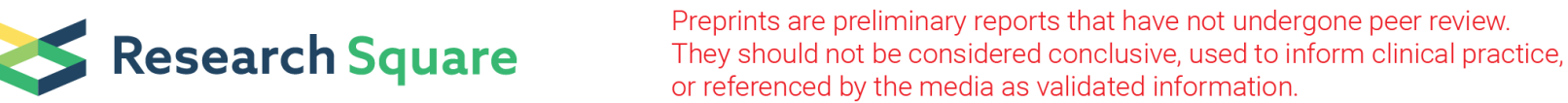

\section{A Chimera Antigen Receptor Containing TLR4 Signaling Domain Enhances CAR-iMACs Polarization and Potency against Solid Tumors}

Anhua Lei

Zhejiang University

Yu Hua

Zhejiang University

Shan Lu

Zhejiang University

Mengmeng Zhu

Zhejiang University

Tianyu Tan

Zhejiang University

Lin Tian

The First Affiliated Hospital and Center for Stem Cell and Regenerative Medicine, Department of Basic

Medical Sciences, Zhejiang University School of Medicine, Hangzhou, Zhejiang, China.

\section{Xudong Wang}

Zhejiang University

\section{Li Zhang}

Zhejiang University

wei zhao

Siyu Su

Fujian Medical University

Hailing Zhang

Zhejiang University

Hengxing Lu

Zhejiang University

\section{Yuqing Zhu}

Zhejiang University

Jing Zhao

Zhejiang University

Wenhong Jiang

Zhejiang University

Chong Liu 
Zhejiang University

Jin Zhang ( $\nabla$ zhgene@zju.edu.cn )

Zhejiang University https://orcid.org/0000-0003-0197-3624

\section{Article}

Keywords:

Posted Date: January 31st, 2022

DOI: https://doi.org/10.21203/rs.3.rs-1279112/v1

License: (c) (1) This work is licensed under a Creative Commons Attribution 4.0 International License. Read Full License 


\section{Abstract}

Chimeric antigen receptor (CAR)-T cell therapies have shown success in treating certain types of hematologic malignancies, but its therapeutic effect on solid tumors is unsatisfactory. Macrophages came to attention because of its phagocytosis function against tumor cells and its immunomodulation capacity. The first generation of engineered CAR-macrophages demonstrated that the CAR can stimulate macrophage phagocytosis function in an antigen dependent way. In this work, we genetically engineered induced pluripotent stem cell (iPSC) derived macrophages (iMACs) with a TLR4 intracellular TIR domaincontaining CAR, and achieved enhanced anti-tumor effect. CD3Z-TIR-CAR, the second generation of TIRbased dual signaling CAR endowed iMACs the target engulfing capacity against antigen-expressing tumor cells, as well as potency of antigen-dependent M1 polarization and resistance to M2 polarization in a NF-KB dependent manner. Taken together, we established the next generation of CAR-iMAC equipped with concurrent antigen-dependent phagocytosis and polarization capacity for better anti-tumor functions.

\section{Introduction}

Cancer immune cell therapies illustrated by CAR-T cells have achieved great success in B cell leukemia/lymphoma and other hematologic malignancies ${ }^{1-7}$. Although the therapeutic cells were designed to precisely target solid tumor antigens, the treatment of solid tumors has not achieved substantial progress ${ }^{8-11}$. To date, the challenges such as exhaustion in the immunosuppressive tumor microenvironment $(\mathrm{TME})^{12,13}$, limited infiltration into dense extracellular matrix ${ }^{14-17}$, off-target effects ${ }^{18}$, antigen escape and heterogeneity within the tumor etc ${ }^{19,20}$, have been thought to be the major obstacles for successful immune cell therapies against solid tumors.

Macrophage, a terminally differentiated monocytic phagocyte, comes to the horizon of tumor immunotherapy field because of its engulfing capacity and central role in the crosstalk between adaptive and innate immune systems ${ }^{21-24}$. Macrophages are found to reside in normal tissues and tumors, and play critical roles in defensing pathogens and combating cancer cells ${ }^{21,23-26}$. As a consequence, macrophages become a potential target of immunotherapy or a type of therapeutic cell for adoptive transfer $25,27-31$. Tumors have been found to consist of large number of immune cells besides neoplastic cells $^{32-34}$. Among the tumor-residing leukocytes, tumor-associated macrophages (TAMs) account for the majority of the population ${ }^{25,35}$. However, these macrophages are reprogramed toward the alternatively activated M2 phenotype, and play tumorigenic role in breast, prostate, ovarian and other carcinomas, through suppressing other immune responses, inducing angiogenesis, etc ${ }^{36-43}$. Therefore, the effort to introduce more pro-inflammatory M1 polarization macrophages state or to convert M2 to M1 macrophages in the immunosuppressive TME would be a viable anti-tumor strategy ${ }^{44-46}$. Previous efforts mainly focused on targeting endogenous macrophages or TAMs ${ }^{47,48}$. Recent technology progress in genetically engineering macrophages enabled us to better harness macrophages as a type of therapeutic cells for adoptive transfer $29,31,49-55$. Currently, pre-clinical and clinical studies using 
macrophages that have been genetically engineered are in development. In consideration of the availability of large source, stability and standardization of the engineered macrophages, iPSCs differentiated iMACs have been utilized as a valuable alternative solution ${ }^{29,56-58}$. Previous works verified the feasibility of producing CAR-iMACs, and demonstrated the CAR-dependent activation of CAR-iMACs in the treatment of solid and blood cancers ${ }^{29}$. However, the 1 st generation of CAR containing CD3Z activating domain used in macrophages was borrowed from CAR-T cells (T-CAR), and was mainly responsible for effector cell function of phagocytosis, and in theory had no capacity to polarize macrophages toward the durable M1 pro-inflammatory state ${ }^{29}$. Thus, it's urgent to design a new macrophage specific CAR (M-CAR) to confer both phagocytosis and polarization functions.

Toll-like receptors (TLRs) are sensors of pattern-recognition receptors (PRRs), and are important players in immune regulation through recognizing distinct pathogen-associated molecular patterns (PAMPs) ${ }^{59-}$ 61. Upon binding with respective PAMPs, TLRs recruit myeloid differentiation factor 88 (MyD88), Toll/IL$1 R(T I R)$-domain-containing adapter-inducing interferon- $\beta$ (TRIF) and other adaptor molecules to initiate activation of downstream signaling including nuclear factor kappa B (NF-kB), and thereby leads to expression of inflammation-associated genes and release of cytokines ${ }^{59,62}$. TLR4, a typical TLR member, is widely distributed in leukocytes, especially myeloid cells such as macrophages ${ }^{63,64}$. Once recognizing lipopolysaccharide (LPS), TLR4 interacts with MyD88 or/and TRIF via its TIR signal transduction domain, and leads to nuclear translocation of NF-kB/p65, which promotes the expression of pro-inflammatory cytokines such as interleukin (IL)-1A, IL-1B, IL-6, IL-12, Nos2, chemokine (C-C motif) ligand 8 (CCL8), and tumor necrosis factor (TNF)- $a^{65-71}$. Based on the biological mechanisms of TLR4, we hypothesized that introducing the intracellular TIR domain of TLR4 into the CAR would conduce to stimulate and maintain the M1 phenotype of iMACs upon engaging antigens, and to elevate anti-tumor efficacy.

In this work, we developed the 2nd generation macrophage-specific CAR (M-CAR) by integrating intracellular CD3 3 and TIR domains in tandem, to construct an EGFRvlll-targeting M-CAR. Genetically modification of iMACs with the 2 nd generation M-CAR markedly improved antigen-dependent anti-tumor efficacy both in vitro and vivo. With further cellular and molecular analysis and comprehensive single cell RNA-seq assessment we proved the underlying mechanisms were that TIR promoted the M1 proinflammatory state and suppressed the M2 state in a NF-KB pathway-dependent manner. Thus, we established a proof-of-concept 2nd generation of CAR-iMACs for future cancer immune cell therapies in solid tumors.

\section{Results}

\section{Construction of macrophage specific CARs and development of EGFRvIll-targeting CAR-iMACs}

The $\mathrm{CD} 3 \zeta$ signal transduction domain obtained from $\mathrm{T}$ cell specific CAR has been proved to be capable of stimulating the first generation of human CAR-iMACs $29,31,49$. However, the domain cannot be helpful to 
maintain the M1 state of macrophages in $\mathrm{TME}^{29}$. Therefore, we designed macrophage specific CARs containing the intracellular TIR domain, whose activation can drive M1 polarization of macrophages via interplaying with TIR domain-containing adaptors. Meanwhile, we employed EGFRvIll-targeting ScFv as the extracellular domain. We designated it as TIR-CAR to distinguish it from the previous CD3Z-CAR (Figure 1a). Inspired by the evolutionary process of different generations of CARs in T cells (T-CARs), we simultaneously designed a second generation macrophage specific CAR (M-CAR) by integrating

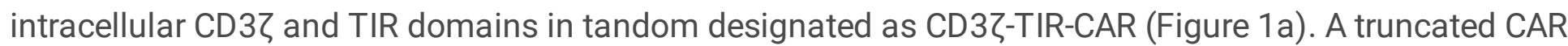
without an intracellular domain was designed as the negative control (Figure 1a). We then transduced the above CARs into human iPSCs with lentivirus. CAR-expressing iPSCs were subsequently differentiated into CAR-iMACs through the periods of mesoderm induction, hematopoietic specification, myeloid expansion, and macrophage maturation (Figure 1b). Flow cytometric (FCM) analysis showed that all types of CAR-iMACs shared about $80 \%$ transgene expression (Figure 1c). Thus, we successfully developed four types of EGFRvIll-targeting CAR-iMACs.

We subsequently analyzed expression of TLR4 signaling pathway genes at different stages of myeloids/macrophages differentiation with RNA-seq data. We found that the key factors such as IRF7, NF-KB/P65, MYD88, TRAP6, TRAM, and TAB2/3 were highly expressed on day 18 and declined on day 28 (Figure 1d). This data suggested that most of the downstream modulators of TLR4 are higher when the iMACs are still in a monocyte-like state, compared with the later stage when the iMACs are more matured. Furthermore, in vitro M1 polarized iMACs expressed higher level of the same TLR4 signaling pathway genes compared to the naive or M2 polarized iMACs (Figure 1e). Together, the data suggested that the early differentiated iMACs resembled the M1 polarized iMACs in terms of higher expression of the TLR4 pathway adaptor and signal transduction genes, and therefore might possess more potent signaling effects from the activated TIR domain.

In order to make a target cell line for the EGFRvIII CAR expressing iMACs, we cloned the human EGFRvIII sequence into a lentivirus vector (Supplemental Figure 1a), and overexpressed the gene in U87MG cells to

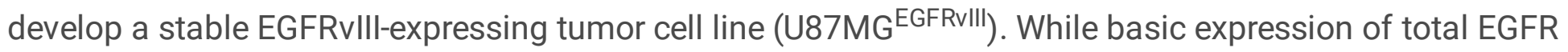
was observed in both wild-type (WT) and transgenic U87MG cells, only the latter had strong expression of EGFRVIII protein (Supplemental Figure 1b and c). With immunofluorescence, we observed the location of EGFRVIII on the cell membrane, as well as in cytoplasm due to overexpression (Supplemental Figure 1d). Thus we have successfully constructed an U87MG EGFRvIll cell line for testing effector functions of EGFRvIII CAR-iMACs.

\section{Both TIR and CD3Z domains enhanced antigen-dependent functions of CAR-iMAC in vitro}

Previous work demonstrated that the first generation of CAR conferred antigen-dependent functions of CAR-iMACs $^{29,31,49}$. In order to evaluate whether the TIR signal domain can also enhance phagocytosis of CAR-iMACs against tumor cells in an antigen-dependent manner, we first performed experiments to incubate the single intracellular domain-containing CAR-iMACs cells with U87MG ${ }^{\text {EGFRvill }}$ cells in vitro. We first stimulated WT-iMACs, truncated CAR-iMACs, CD3Z-CAR-iMACs, and TIR-CAR-iMACs with IFN- $y$ and 
LPS respectively, and then co-cultured the cells with tdTomato-expressing (tdTomato ${ }^{+}$) U87MG EGFRvIII cells with different effect/target ratios $(E / T)$ of $3 / 1,5 / 1$, and 10/1. After 12 hours of co-culturing, we observed enhanced adhering property of CAR-iMACs to the antigen-expressing cancer cells compared to WT-iMACs (Supplemental Figure 1e), signifying that the CAR enabled iMACs with targeting capacity. Moreover, immunofluorescence assays showed that both CAR-iMACs and WT-iMACs engulfed tdTomato labeled tumor cells (Supplemental Figure 1f), demonstrating that the iMACs possess basic phagocytosis like primary macrophages, and overexpression of CAR did not impair this feature. Next, we collected all the cells and assessed the number of residual tumor cells by FCM analysis (Supplemental Figure 2a). Compared to WT-iMACs or truncated CAR-iMACs, U87MG ${ }^{\text {EGFRvill }}$ cells co-incubated with CD3ろ or TIR-CARiMACs suffered more killing and remained fewer cells at the same condition of $E / T$ ratio (Supplemental Figure 2a and $b$ ). At the same time, all types of CAR-iMACs showed strengthened cytotoxicity with increased E/T ratios (Supplemental Figure $2 b$ ). Moreover, we did not observe obvious disparity of cytotoxicity between CD3Z-CAR-iMACs, and TIR-CAR-iMACs at the 12 hours incubating time point (Supplemental Figure 2b).

To demonstrate progressive cytotoxicity of CAR-iMACs against their target cells, we next performed the same experiments at 24 hours of co-incubating. Compared to 12 hours, 24 hours co-incubating significantly improved tumor cell killing efficacy of CAR-iMACs at all tested E/T ratios (Figure 2a and b). Although both CD3Z-CAR-iMACs and TIR-CAR-iMACs showed powerful anti-tumor competence compared to truncated iMACs, it appeared the TIR domain conferred stronger killing capacity than $\mathrm{CD} 3 \zeta$ at this time point (Figure 2b). When we compared the trend of killing effect of the three types of CAR-iMACs from 12 hours to 24 hours co-incubating with U87MG ${ }^{\text {EGFRvIll }}$ cells, we found that TIR-CAR-iMACs had greater killing persistence than CD3Z-CAR-iMACs as time goes on (Figure 2C). To validate the above findings, we overexpressed a firefly luciferase (FFluc) gene in U87MG EGFRvill cells, and performed more killing assays to further test the anti-tumor cell capacity of the CAR-iMACs. Consistently, the bioluminescence signal from tumor cells was evidently attenuated by both CD3Z-CAR-iMACs and TIR-CAR-iMACs comparing to truncated CAR-iMACs, and the efficiency achieved optimum at the E/T ratio of 10/1 (Figure 2d). Furthermore, in order to investigate whether the pro-inflammatory activity of CAR-iMACs is relied on CAR activation, we measured release of TIR downstream cytokines. ELISA tests revealed that when encountering U87MGEGFRvIll cells for 24 hours at an E/T ratio of 10/1, TIR-CAR-iMACs secreted considerable pro-inflammatory cytokines including IL6 (Figure 2e), IL12A (Figure 2f) and IL23 (Figure 2g), indicating more pro-inflammatory activity of TIR-CAR-iMACs. Interestingly, the CAR containing T cellspecific $\mathrm{CD} 3 \zeta$ domain also promoted milder expression of these cytokines in CAR-iMACs (Figure 2e-g), and the expression of TNF- $a$, another TLR4 regulated immune factor, was unexpectedly higher in CD3 $\zeta$ CAR-iMACs than in TIR-CAR-iMACs (Figure $2 h$ ), suggesting combining the two might achieve the maximal pro-inflammatory activity. Taking together, these results demonstrated that both TIR and CD3Z domains enhanced CAR-dependent cytotoxic activity of CAR-iMACs, but TIR-CAR-iMACs exhibited more potential of persistent and potent pro-inflammatory activity.

TIR-CAR-iMACs possess stronger anti-tumor potency than CD3Z-CAR-iMACs in vivo 
We next investigated anti-tumor activities of CD3Z-CAR-iMACs and TIR-CAR-iMACs in tumor-bearing animals. We first established the tumor model in abdominal cavity of immunodeficient mice with FFluc ${ }^{+}$U87MG ${ }^{\text {EGFRvIll }}$ cells. IFN-y/LPS polarized truncated CAR-iMACs, CD3Z-CAR-iMACs, and TIR-CARiMACs were labeled by the DiR dye, and then injected intraperitoneally into the tumor-bearing mice with an E/T ratio of 10/1. Animal imagings were performed 2 hours after immune cells injection (day 0 ), and at the same time of day 1, day 3 and day 7 (Figure 3a). All the three types of CAR-iMACs exerted anticancer cell activity against the tumor at day 1 immediately (Figure $3 b$ and $c$ ). However, comparing with truncated CAR-iMACs and CD3Z-CAR-iMACs, TIR-CAR-iMACs exhibited stronger anti-tumor activity, and achieved optimum efficacy at day3 (Figure 3c). Of note, even though the tumor started to relapse at day 7, the tumor signals from TIR-CAR-iMACs-treated mice were still maintained at a lower level than that in PBS and CD3Z-CAR-iMACs-treated mice, suggesting that introducing the TIR domain into CAR-iMACs might have conferred resistance to reprogramming by the tumor microenvironment over time. Further survival analysis showed that TIR-CAR-iMACs markedly prolonged the life time of tumor-bearing mice (Figure 3d). Collectively, the data demonstrated that although both TIR-CAR-iMACs and CD3Z-CAR-iMACs showed antigen-dependent anti-tumor activity in vivo, the TIR-CAR conferred more persistent killing capacity of CAR-iMACs against cancer cells.

\section{The combination of TIR and CD3ろ domains strengthened M1 polarization and anti-tumor potency of CAR- iMACs}

In the above work, we have verified that TIR-CAR-iMACs exhibited the M1 phenotype and both TIR and CD3ろ domains enabled CAR-iMACs with antigen-dependent phagocytosis capacity. Based on the design of second generation of T-CAR, we hypothesized that integration of TIR and CD3 3 domains into one CAR structure could further promote anti-tumor potency of CAR-iMACs. Thus we developed a new M-CAR

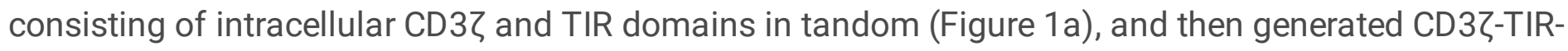
CAR-iMACs (Figure 1C). We first compared expression of pro-inflammatory or M1 polarization genes supposedly driven by TIR activation. As expected, qPCR analysis revealed that, when incubating the three types of CAR-iMACs with U87MGEGFRvIll cells for 24 hours, expression of IL 1A/B, IL6, IL 12A, IL23, CCL8, CXCL8 and TNF- $a$ were elevated significantly in CD3Z-TIR-CAR-iMACs compared to TIR- or CD3Z-CARiMACs at the condition of no IFN-y/LPS pretreatment (Figure 4a-h), revealing a synergistic effect of TIR and $\mathrm{CD} 3 \zeta$ domains. Correspondingly, ELISA testings confirmed that CD3Z-TIR-CAR-iMACs improved production of IL6, IL12A, IL23, and TNF-a (Figure 4i-I). These results further demonstrated that the TIR domain contributed to M1 polarization of CAR-iMACs. Subsequently, we examined cytotoxicity of the integrated CD3Z-TIR-CAR-iMACs confronting FFluc ${ }^{+}$U87MG ${ }^{\text {EGFRvlll }}$ cells. Measurement of bioluminescence from FFluc ${ }^{+} U 87 M M^{E G F R v l l l}$ cells indicated that comparing to single CD3 3 or TIR domain-containing CARiMACs, 24 hours incubation with CD3ろ-TIR-CAR-iMACs led to the lowest number of residual tumor cells under the condition of E/T of 10/1(Figure $4 \mathrm{~m}$ ), revealing that CD3ろ-TIR-CAR-iMACs conferred much more lethality against EGFRvIll positive tumor cells than single domain-containing CAR-iMACs. 
Previous reports have illustrated that the TME leads to exhaustion of T cells and promotes M2-like polarization of macrophages ${ }^{12,38,72-75}$. To explore whether the TIR based CAR-iMACs were equipped with the ability of withstanding immunosuppressive effects from tumor cells, we examined the polarization state of the four types of CAR-iMACs co-cultured with tdTomato ${ }^{+}$U87MG ${ }^{\text {EGFRvill }}$ cells under the condition of no IFN- $y / L P S$ pre-treatment and an E/T ratio of 3/1. Subsequent FCM analysis showed that 24 hours incubation with the tumor cells stimulated high expression of CD80 in all types of CAR-iMACs, and the two TIR-based CAR-iMACs showed more CD80-positive $\left(C D 80^{+}\right)$populations compared to those in truncated CAR-iMACs and CD3Z-CAR-iMACs (Supplemental Figure 3a). Of note, prolonged periods of exposure to tumor cells resulted in marked reduction of $\mathrm{CD} 80^{+}$populations to about $40-50 \%$ for truncated CAR-iMACs and CD3Z-CAR-iMACs (Supplemental Figure 3a, and Figure $4 \mathrm{n}$ and $\mathrm{p}$ ), whereas the percentage of $\mathrm{CD} 80^{+}$cells in both TIR-CAR-iMACs and CD3Z-TIR-CAR-iMACs persisted at a higher level of $~ 80 \%$ (Supplemental Figure 3a, Figure $4 \mathrm{n}$ and $\mathrm{p}$ ), indicating that TIR contributed to stimulation of the M1 state. On the other hand, we also determined M2 populations of the four types of CAR-iMACs by measuring CD163, a typical M2 marker, and found that long time exposure to the tumor cells led to various level of CD163 expression in the first three types of CAR-iMACs, whereas CD3Z-TIR-CAR-iMACs exhibited robust potency against M2 polarization (Supplemental Figure 3b, Figure 40 and q). Indeed, the percentage of CD163 ${ }^{+}$CD3Z-TIR-CAR-iMACs declined to about $36.93 \%$ at day 3 of co-incubation, and remained to be low at day 7 (Supplemental Figure $3 \mathrm{~b}$ and Figure 40 and $\mathrm{q}$ ). Based on the above results, we concluded that the combination of the TIR and CD3Zdomains supported M1 polarization of CAR-iMACs exposing to tumor cells, and contributed to prevent reversion of M1 to M2 state.

We next investigated anti-tumor potency of CD3ろ-TIR-CAR-iMACs in vivo. Operated as above, truncated

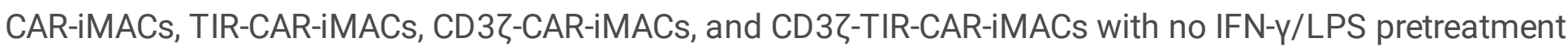
were injected intraperitoneally into FFluc ${ }^{+} U 87 M^{E G F R v I l l}$ cells-bearing mice at the condition of an E/T ratio of 15/1 (Supplemental Figure 4a). Bioluminescence imagings were performed at day 0 , day 1 , day 3 , and day 7. As expected, treatment by TIR-CAR-iMACs or CD3Z-TIR-CAR-iMACs markedly alleviated tumor cell growth at day 1 compared to truncate CAR-iMACs or CD3Z-CAR-iMACs (Supplemental Figure 4b and c). Noteworthy, CD3Z-TIR-CAR-iMACs showed the strongest anti-tumor potency in the living body environment (Supplemental Figure $4 \mathrm{~b}$ and $\mathrm{c}$ ), and prolonged survival time of the tumor-bearing mice most prominently (Supplemental Figure 4d). In order to assess whether TIR contributed to M1 polarization of CAR-iMACs in the course of treatment inside the living body, we isolated the immune cells from abdominal cavities of the mice after two days of treatment, and measured $C D 80^{+}$and CD $163^{+}$populations by FCM. As observation in vitro, more TIR-CAR-iMACs and CD3Z-TIR-CAR-iMACs exhibited the CD80 positive feature than that in CD3Z-CAR-iMACs (Supplemental Figure 4e and f), and much lower CD163 positive M2 feature was present in CD3Z-TIR-CAR-iMACs (Supplemental Figure 4e and f). In summary, we confirmed that integration of intracellular CD3 3 and TIR domains into one CAR structure could enhance cytotoxic activity against tumor cells and potency of maintaining M1 polarization of CAR-iMACs. 
The CAR promotes M1 polarization of CAR-iMACs via TIR domain-mediated canonical NF-kB/P65 transcription activity

The above data demonstrated that the TIR domain enhanced M1 polarization and activation of CARiMACs in an antigen-dependent manner. TIR domain is responsible for signal transduction from TLR4, and the downstream signaling liberates NF-KB/P65 from IKB-a-mediated degradation followed by the translocation of NF-KB/P65 into nucleus to transcriptionally activate pro-inflammatory target genes ${ }^{70,76}$. In order to investigate whether NF-KB/P65 mediated the pro-inflammatory activity of TIR-CAR-iMACs encountering the antigen, we first tested expression of $N F-K B / P 65$ gene RELA with qPCR analysis and found its higher expression in both TIR domain-containing CAR-iMACs (Figure 5a). Whereas ERK, another gene involved in macrophage activation had no distinction of expression in all CAR-iMACs (Figure $5 b$ ). Next, we monitored nucleus translocation of NF-KB/P65 by immunofluorescence when the control truncated CAR-iMACs were treated with LPS for 20 minutes (Supplemental Figure 5a). Afterwards, we co-

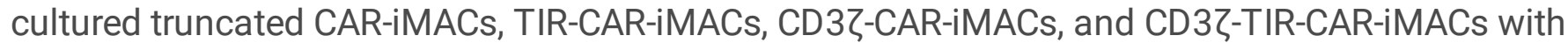
tdTomato ${ }^{+}$U87MG ${ }^{E G F R v I l I}$ cells respectively with an E/T ratio of $3 / 1$, followed by immunoflurescence to detect NF-кB/P65 subcellular localization. Confocal imaging showed that sufficient contact between CAR-iMACs and U87MGEGFRvIII cells for 4 hours initiated expression of NF-KB/P65 in CD3ろ-TIR-CAR-iMACs (Supplemental Figure 5b). When the incubating time was prolonged to 10 hours, NF-KB/P65 exhibited evident expression in both TIR-CAR-iMACs and CD3Z-TIR-CAR-iMACs, and weak expression in CD3Z-CARiMACs, and it aggregated in the nucleus of the two types of TIR-based CAR-iMACs (Figure $5 \mathrm{c}$ and d). Nevertheless, after 24 hours of incubation, the aggregation of NF-KB/P65 in the nuclei of TIR-CAR-iMACs or CD3Z-TIR-CAR-iMACs was abrogated (Supplemental Figure 5c), resembling the outcome of LPSinduced tolerance in macrophages ${ }^{77-81}$. Notably, phagocytosis of tumor cells also led to nuclear export and cytoplasmic accumulation of NF-KB/P65 in TIR-CAR-iMACs and CD3Z-TIR-CAR-iMACs (Supplemental Figure $5 \mathrm{c}$ ), indicating inactivation of the immune cell functions after completing tumor-killing process. Besides, we didn't detected obvious expression and nuclear localization of NF-KB/P65 in the TIR-CARiMACs that did not touch U87MG EGFRvill cells (Figure 5c), consistent with an antigen-dependent activation mechanism. Together, these results demonstrated that the NF-KB signal pathway was activated in the TIR domain-containing CAR-iMACs.

To further confirm the role of NF-KB/P65 in CAR-iMAC, we treated the CAR-iMACs co-cultured with FFluc/tdTomato ${ }^{+}$U87MG ${ }^{\text {EGFRvIll }}$ cells with a NF-KB/P65 inhibitor JSH23. As expected, blocking of nuclear location of NF-KB/P65 by JSH23 abrogated cytotoxicity of CAR-iMACs against FFluc/tdTomato ${ }^{+}$ U87MG ${ }^{\text {EGFRvIll }}$ cells (Figure 5e). Similarly, FCM analysis of residual tumor cells indicated that blocking NFKB/P65 into nucleus increased residual tdTomato positive tumor cells in TIR-CAR-iMACs and CD3Z-TIRCAR-iMACs groups (Figure 5F). Collectively, we confirmed that immune activation and M1 polarization of TIR-based CAR-iMACs were through the NF-KB pathway.

\section{Single cell RNA-sequencing revealed robust molecular features and mechanisms of M1 polarization of TIR-based CAR-iMACs}


We next attempted to examine to what extent the TIR-based CAR-iMACs could promote the proinflammatory state when exposed to tumor cells through single cell RNA-sequencing analysis (Figure 6a). In this experiment, we pretreated the CAR-iMACs with IFN-y/LPS for 24 hours, and then incubated each of them with U87MG EGFRvill cells for another 24 hours. After that, we performed 10xgenome single cells RNAsequencing analysis with all the cells, and found that they could be clustered into six sub-populations (Supplemental Figure 6a, $\mathrm{c}$ and e). According to the in vivo single cell RNA-seq data of human hematopoietic development, $\mathrm{C} 1, \mathrm{C} 2$ and $\mathrm{C} 3$ clusters were mainly matched to the Macrophage cell phenotype (Supplemental Figure $6 \mathrm{~b}, \mathrm{~d}$ and $\mathrm{f}$ ). Meanwhile, $\mathrm{C} 4$ and $\mathrm{C} 5$ clusters were primarily matched with the U87MG cancer cell phenotype in all the three types of CAR-iMACs groups we examined. Interestingly, the $\mathrm{C} 5$ cluster also showed evident macrophage feature in the CD3Z-TIR-CAR-iMACs group, indicating these were the cells that engulfed the tumor cells and thus assumed features of both macrophage and tumor cells (Supplemental Figure 6b, $d$ and $f$ ). According to the single cell analysis, compared with truncated CAR-iMACs and TIR-CAR-iMACs groups, CD3ろ-TIR-CAR-iMACs treatment resulted in fewer remaining tumor cells (Supplemental Figure 6a-f, and Figure 6b). In addition, even though the C6 cluster of all the three groups were mainly matched with macrophage, $\mathrm{C} 6$ in TIR-CAR-iMACs and CD3Z-TIR-CARiMACs showed more evident and incremental DC characteristics compared to truncate CAR-iMACs (Supplemental Figure $6 b, d$ and $f$ ), implying that TIR-based CAR-iMACs might have acquired enhanced antigen-presenting competence as dendritic cells.

As in the above results, we have demonstrated the M1 polarization-promoting effect of TIR-CAR. Next, we performed more quantitative analysis of M1 or M2 markers using single cell RNA-seq data. Normalized gene expression showed that more TIR-CAR-iMACs exhibited high expression of $C D 80$ and $C D 86$, and

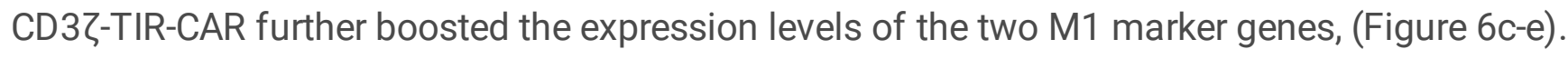
Accordingly, expression of M2 marker CD206 was reduced in TIR-CAR-iMACs, and to the lowest level in CD3Z-TIR-CAR-iMACs, compared to that in truncated CAR-iMACs (Figure 6c-e). Moreover, the percentage of CAR-iMACs that expressed other M1 state-associated genes including CD83, CCL8, CXCL9, and CXCL11, increased incrementally from truncated CAR-iMACs to TIR-CAR-iMACs and CD3ろ-TIR-CAR-iMACs (Figure 6f). Accordingly, the normalized expression level of these genes exhibited the same trend (Figure 6g-j). On the contrary, fewer percentage of TIR-CAR-iMAC and CD3Z-TIR-CAR-iMACs expressed M2 stateassociated genes such as CCL13 and CD163 (Figure 6k), and even though only a handful of cells had these genes being detected, TIR-CAR-iMAC and CD3ろ-TIR-CAR-iMACs presented much lower expression compared to truncated CAR-iMACs (Figure $6 \mathrm{I}$ and $\mathrm{m}$ ). Collectively, all the results illustrated that integration of the TIR and $\mathrm{CD} 3 \zeta$ domains facilitated M1 genes expression and suppressed M2 gene expression in the same time.

Next we further investigated whether the global gene expression signature resembled that of the M1 macrophages. We divided CAR-iMACs to CD80-expressing and CD80-absent (non-CD80) populations, as the latter represented the iMACs that might not encounter tumor cells, and evaluated the discrepancy of their expression profile. Principle component analysis (PCA) using M1-related genes identified in our previous study indicated that CD80-expressing TIR-CAR-iMACs and CD3Z-TIR-CAR-iMACs were positioned 
closer to the IFN-y/LPS-stimulated M1 iMACs ${ }^{29}$, compared to CD80-positive truncated CAR-iMACs and non-CD80 CAR-iMACs from all the types (Figure 6n). Furthermore, pathway analysis revealed that CD80expressing CAR-iMACs showed lower level of M2-related pathways such as Oxidative phosphorylation and Respiratory electron transport, than that in non-CD80 CAR-iMACs, but higher level of M1-related pathway Interferon gamma response (Figure 60). More importantly, within the CD80-expressing groups, TIR-CAR-iMACs and CD3Z-TIR-CAR-iMACs showed evidently higher Interferon gamma response and lower Oxidative phosphorylation (Figure 60), indicating that TIR based CAR conferred the CAR-iMACs with strengthened pro-inflammatory activity. Lastly, via hierarchical clustering analysis based on a defined set of M1 and M2-associated genes ${ }^{29}$, we found that both TIR-CAR-iMACs and CD3Z-TIR-CAR-iMACs clustered with IFN-y/LPS- polarized iMACs, whereas in contrast, truncated CAR-iMACs clustered with M2 polarized iMACs (Supplemental Figure 7a and $b$ ). The same trend of clustering results was observed when using the CD80-expressing populations (Supplemental Figure 7c and d). To sum up, the above single cell RNA-seq analysis strongly demonstrated that TIR-based CARs contributed to the M1 polarization of CAR-iMACs in an antigen-dependent manner when encountering tumor cells, and improved immune activity against target tumor cells.

\section{Discussion}

Recently, the macrophage and genetically engineered CAR-macrophage have drawn lots of attention because of its potential advantages in homing and infiltrating to solid tumor, and manifold roles in regulating the immunosuppressive tumor microenvironment ${ }^{36,82}$. More excitingly, the first clinical study in patients with solid tumor has started ${ }^{83}$, and the proof-of-concept results will justify CAR-macrophage as a new weapon to combat solid tumor.

The above achievements are all based on the 1st generation of CAR-macrophage, in which the intracellular signaling is mediated by a $\mathrm{CD} 3 \zeta$ domain or a substitute effector domain from macrophage $29,31,49,84,85$. Like the 1 st generation of CAR in T cells, this CAR mainly confers the effector function of phagocytosis, or the "signal 1" for CAR-macrophages. In order to integrate a "signal 2" like the co-stimulatory CD28 or 4IBB intracellular domain (ICD) in CAR-T ${ }^{1,86-92}$, we added a TLR4 intracellular domain TIR into the CAR, and thus provided an orthogonal "signal 2" of macrophage polarization. We demonstrated that the iPSC-derived, 2nd generation of EGFRvIII CD3Z-TIR-CAR-macrophage against antigen-expressing tumor cells exhibited enhanced anti-tumor activity both in vitro and in vivo, with a particularly strong M1 polarization phenotype.

It is well established that macrophages are equipped with receptors called pattern recognition receptors (PRRs) that recognize "non-self" pathogen- or damage-associated molecular patterns (PAMP or DAMP), and then release pro-inflammatory or anti-inflammatory cytokines to exert various functions in defense ${ }^{59,65}$. The TIR-containing chimera antigen receptor is a novel engineered PRR which recognizes "antigen-associated molecular patterns", and enables macrophage with antigen-dependent capacity of polarization, in this case to be more pro-inflammatory to aid immune cell therapies in cancer. The

Page $11 / 36$ 
opposite way of anti-inflammation can also be exploited to combat autoimmune diseases. These novel engineered macrophages with $T$ cell and $B$ cell function modules further blur the boundary between different immune cell types, and with the iPSC platform of high engineering feasibility, more and more payloads can be added onto this synthetic immune cell type to achieve better anti-tumor or other functions.

\section{Methods}

Cell culture. Human iPSCs were cultured in mTeSR medium (STEMCELL Technologies, \#85851) with Matrigel Matrix (Corning, \#354277) coated 6-well cell culture plate. iPSCs were passaged using Versene (Gibco, \#15040-066). Matured CAR-iMACs and WT-iMACs were maintained in StemSpanXF medium (STEMCELL Technologies, \#100-0073) containing M-CSF $(100 \mathrm{ng} / \mathrm{mL})$ and GM-CSF $(100 \mathrm{ng} / \mathrm{mL})$. 293T cells were cultured in high-glucose DMEM (Gibco, \#11965-065) containing 10\% FBS (Gibco, \#10099-141), and $1 \times$ Pen Strep (10,000 U/ml penicillin, 10,000 $\mu \mathrm{g} / \mathrm{ml}$ streptomycin) (Gibco, \#15140-122). U87MG cells and U87MG ${ }^{\text {EGFRvIll }}$ cells were cultured in MEM (Hyclone, \#SH30024.01) medium supplemented with 10\% FBS (10099-141, Gibco), 1×Non-Essential Amino Acids (Gibco, \#11140-050), 1mM Sodium Pyruvate (Gibco, \#11360-070), and 1×Pen Strep (10,000 U/ml penicillin, 10,000 $\mu \mathrm{g} / \mathrm{ml}$ streptomycin) (Gibco, \#15140-122).

Construction of CAR. We designed T-CAR or M-CARs according to the intracellular CD3 3 domain from T cell receptor or TIR domain derived from TLR4, integrating hinge and transmembrane region of human CD8a (amino acids 183-206). The intracellular fragment of the CAR is humanized scFv nucleotide sequence specific to EGFRvIII (139 scFv nucleotide sequence. Patent No.: US 9,394,368 B2). Cytoplasmic part of the human CD3ろ (amino acids 52-164) was designed for T-CAR. The TIR domain derived from cytoplasmic portion of human TLR4 (amino acids 672-818) was designed for M-CAR. The integration of cytoplasmic parts of the $\mathrm{CD} 3 \zeta$ and TIR were designed for the second generation M-CAR. All the CARs were synthesized by GenScript Biotech, and cloned into the EcoRI and BamHI sites of Lenti-EF1A-T2AEGFP-Puro vector.

Stable overexpression of CARs in iPSCs. CAR sequence-containing lentiviral vector, psPAX2, and pMD2.G were transfected into HEK293T cells cultured in $10 \mathrm{~cm}$ dishes to produce Lentivirus using Lipofectamine 2000 (Invitrogen, \#11668). Medium was removedafter 6 hours of transfection to remove transfection reagent. Afterwards, lentivirus containing medium was harvested at the time point of $24 \mathrm{~h}, 36 \mathrm{~h}, 48 \mathrm{~h}$, ad $60 \mathrm{~h}$ post transfection, and filtered with a $0.45 \mu \mathrm{m}$ filter. The filtered medium was mixed with half volume of $30 \%$ PEG8000, and concentrated at $4000 \mathrm{rpm}$ for $30 \mathrm{~min}$ at $4^{\circ} \mathrm{C}$. The supernatant was discarded, and the pellet was resuspended with mTeSR medium (STEMCELL Technologies, \#85851) containing 10ug/ml polybrene (Yeasen Biotech, \#40804ES76). The resuspended lentivirus was then added to prepared iPSCs in 6 well plate. The medium was refreshed after 6 hours post infection with new mTeSR medium. The infected cells were cultured for 24 hours, and then treated with $0.5 \mu \mathrm{g} / \mathrm{ml}$ puromycin for at least 48 hours to select successfully transduced clones. Subsquently, the CAR-expressing iPSCs were then expanded. 
Macrophage differentiation from iPSC. WT-iPSCs and CAR-expressing iPSCs were treated with TrypLE (Gibco, \#12604-021) for 1 min to disaggregate into individual cells. The cells werer collected and transferred to low-attachment plates (Corning, \#3471) to allow formation of embryoid bodies (EBs) in mTeSR medium (STEMCELL Technologies, \#85851) supplemented with Rock inhibitor Y27632 (STEMCELL Technologies, \#72304). The following process of formation of embryoid bodies and macrophages differentiation from embryoid bodies has been described in our previous work.

Construction of genetic tumor cells. We first synthesized coding sequence of EGFRvIll in GenScript Biotech, and then cloned the sequence into the Lenti-EF1a-T2A-EGFP-Puro vector. Subsequently, lentivirus containing EGFRvIll coding sequence was harvested from 293T cells, and it was used to infect U87MG cells to develop the U87MG ${ }^{\text {EGFRvIll }}$ cell line.

We over expressed firefly luciferase (FFluc) and tdTomato genes in U87MGEGFRvIll cells via PCDH-EF1atdTomato-FFluc2 plasmids to develop tdTomato/ FFluc ${ }^{+}$U87MG ${ }^{\text {EGFRvill }}$ cell line.

Examination of cytotoxicity of CAR-iMACs against antigen-expressing tumor cells. We first co-cultured EGFP-expressing CAR-iMACs with FFluc ${ }^{+} U 87 M^{E G F R v I l l}$ cells in the case of E/T ratio of 3/1,5/1 and 10/1 respectively for 24 hours in a black 96-well plate (Wohong Bio, \#WHB-96-02) in vitro. We then added 50ul $15 \mathrm{mg} / \mathrm{mL}$ luciferin buffe (GOLDBIO, \#LUCK-1G), and captured biotiniluminescence signal through microplate reader (Molecular devices, \#MD M5).

Detecting of cytokines. We detected the cytokines released by CAR-iMACs with ELISA Kit (Elabscience). After incubating CAR-iMACs with U87MG ${ }^{\text {EGFRvlll }}$ cells for 24 hours, the medium was collected and then centrifuged with $1000 \mathrm{rpm} / 3 \mathrm{~min}$ condition in room temperature. Supernatants were harvested and used to test IL-6 (\#E-EL-H0102c), IL12A (\#E-EL-H0150c), IL23 (\#E-EL-H0107c), and TNF-a (\#E-EL-H0109c). The fluorescence signals were detected by a microplate reader (Molecular devices, \#MD M5).

In vivo anti-tumor assay. Four-weeks old NOD.CB17Prkdc ${ }^{\mathrm{SCID}} / \mathrm{L} 12 \mathrm{fg}^{\mathrm{rm} 1} / \mathrm{Bcgen}$ (B-NDG) mice were obtained from Jiangsu Biocytogen Co., Ltd., and maintained under pathogen-free conditions in the animal centre of Zhejiang Academy of Medical Sciences with Committee-approved protocols. $5 \times 10^{5}$ or $4 \times 10^{5}$ firefly luciferase (FFluc ${ }^{+}$) gene-expressing U87MG ${ }^{\text {EGFRvlll }}$ cells mixed with $0.1 \%$ BSA in phosphate buffered saline (PBS) were injected intraperitoneally into B-NDG mice. Two hours after the tumor cells injection, $5 \times 10^{6}$ or $6 \times 10^{6}$ DiR (Meilunbio, \#MB12482)-dyed CAR-iMACs (Truncated CAR-iMACs, CD3 CAR-iMACs, TIR-CAR-iMAC, and CD3ZETA-TIR-CAR-iMACs) were injected into the same position in abdominal cavity of tumor burdened mice after being mixded with $0.1 \%$ BSA contained PBS. Another two hours after CAR-iMACs injection, D-luciferin (GOLDBIO, \#LUCK-1G) was injected intraperitonelly into the mice with $150 \mathrm{mg} / \mathrm{kg}$ dosage, following with living imaging through animal imaging system (in-vivo Xtreme) to capture the bioluminescence signal. Following animal imaging was conducted at the same time point on day 1, day 3 and day 7 to monitor the tumor growth. During tumor therapy by CAR-iMACs, the survival times of the mice was recorded, and statistics of survival curves was determined. 
Immunofluorescence. The immunofluorescence (IF) assay was conducted to detect expression of EGFRvIII in U87MG ${ }^{\text {EGFRvIll }}$ cell line. After being infected by lentivirus expressing plasmid Lenti-EF1aEGFRvIII-T2A-Puro, U87MG cells were passaged to $0.1 \%$ gelatin coated glass slides in 24 -well cell culture plate. When the cells grew to cover about $80 \%$ of the area of well, discarding mediums and washing the cells with PBS slightly thrice. Afterwards, fixing the cells with 4\% paraformaldehyde (PFA) for 30 minutes, and then discarding the PFA. Repeating the operation three times. Cells were then permeabilized with $0.5 \%$ Triton X-100 for 30 min at room temperature. Subsequently, cells were incubated with primary antibody against EGFRvIII (CST, \#D6T2Q. 1:200) at $4^{\circ} \mathrm{C}$ overnight. Discarding the primary antibody and Washing the cells with PBS slightly thrice followed with incubating the cells with secondary antibody (Alexa Fluor ${ }^{\circledR} 647$ donkey anti-rabbit IgG. abcom, ab150075. 1:1,000) for 1 hour in $37^{\circ} \mathrm{C}$. Afterwards, the glass slides were mounted with anti-fading mounting medium (with DAPI) (Solarbio, \#S2110). Cells were detected by Zeiss LSM800 fluorescence microscope at a 63×oil objective. LSM800 with the Airyscan module was used to capture high-resolution pictures.

For detecting of NF-KB/P65, we first co-incubated EGFP-marked CAR-iMACs with tdTomato+U87MGEGFRvIII cells for $4 \mathrm{hrs}, 10 \mathrm{hrs}$ and $24 \mathrm{hrs}$ respectively in $0.1 \%$ gelatin coated glass slides in a 24-well cell culture plate. Afterwards, processing the slides as described above, and incubating the cells with Alexa Fluor ${ }^{\circledR} 647$ conjugated rabbit antibodies against NF-KB/p65 (CST, \#D14E12. 1:100) at $4^{\circ} \mathrm{C}$ overnight. After being washed with PBS slightly thrice, the glass slides were mounted with anti-fading mounting medium (with DAPI) (Solarbio, \#S2110). Cells were detected by Zeiss LSM800 fluorescence microscope at a $63 \times$ oil objective. LSM800 with Airyscan module was used to capture high-resolution pictures.

RNA isolation and quantitative real-time PCR (qRT-PCR). For the qRT-PCR, total RNA extraction was performed using Eastep Super Total RNA Extraction Kit (Shanghai Promega Bio, \#LS1040) according to the manufacturer's descriptions. cDNA was prepared using HiScript II Q Select RT SuperMix for qPCR with gDNA wiper (Vazyme, \#R223-01). Gene expression was analyzed in triplicate using HiScript II One Step qRT-PCR SYBR Green Kit (Vazyme, \#Q221-04) and Bio-Rad PCR machine (CFX-96 Touch). The difference in cycle threshold values $(\triangle \mathrm{CT})$ of all the genes tested were normalized to the $\triangle \mathrm{CT}$ of GAPDH (glyceraldehyde-3-phosphate dehydrogenase), and the fold change in expression was expressed relative to WT iMACs. The primer sequences used in qRT-PCR assay were listed in Supplemental table.

Immunoblotting (IB). The IB was performed with conventional SDS-PAGE. Wild-type and EGFRvIIIexpressing lentivirus infected U87MG Cells were collected and washed twice with PBS, followed with lysing by RIPA buffer (Beyotime, \#P0013K) containing protease inhibitor (Roche, \#04693124001) on a shaker at $4^{\circ} \mathrm{C}$ for 20 minutes. Cellular debris was removed by centrifugation at $4^{\circ} \mathrm{C}$. The supernatant was harvested and mixed with protein loading buffer that contains SDS, and heated at $90-100^{\circ} \mathrm{C}$ for 10 minutes. Proteins were resolved by SDS-PAGE and transferred to polyvinylidene difluoride membranes. The antibodies used in these experiments were listed as the following: rabbit anti-human EGFR (CST, \#4267. 1:2000), rabbit anti-human EGFRvIII (CST, \#64952. 1:2000), and rabbit anti-human $\beta$-actin (CST, \#4970. 1:10000). Blots were incubated with SuperSignal West Pico PLUS Chemiluminescent Substrate (Thermo Scientific, \#34577) for signal detection.

Page $14 / 36$ 
Fluorescence-activated cell sorting (FACS) analysis. To verify the cytotoxicity of CAR-iMACs against tumor cells, we incubated EGFP-marked CAR-iMACs with tdTomato ${ }^{+}$U87MG ${ }^{\text {EGFRvlll }}$ cells in the case of E/T ratio of $3 / 1,5 / 1$ and 10/1 for 10 hours and 24 hours respectively in vitro. The cells were digested using $0.25 \%$ trypsin-EDTA (meilunbio, \#MA0233). The dissociated cells were resuspended with $0.1 \% \mathrm{BSA}$, and then filtered into individual cells using a 300-mesh. Flow cytometry was performed on Beckman CytoFLEX LX (Version 9). The PE fluorescence channel was chosen to detect tdTomato+ U87MG EGFRvIII cells. The FITC channel was used to recognize EGFP-marked CAR-iMACs. FACS data was collected using CytExpert (Version 2.3), and was processed using FlowJo (Version 9).

To make sure the dynamic change of polarization state of the CAR-iMACs in the presence of tumor cells. We first incubated EGFP-marked CAR-iMACs with U87MGEGFRvIII cells in the case of E/T ratio of 3/1 for 1 day, 2 days, 3 days and 7 days respectively in vitro. After that, we collected the cells as performed as above, and equally divided them into two groups. Cells from one group were dyed with PE fluorophore conjugated antibodies recognizing human CD80 protein (PE-CD80) (Biolegend, \#305208). Another group of cells was labelled with human CD163 specific antibody conjugated with PE (PE-CD163) (Biolegend, \#333605). Then Flow cytometry was performed to detect $C D 80^{+}$and $C D 163^{+}$cells on Beckman CytoFLEX LX. The FITC channel was used to recognize EGFP-marked CAR-iMACs. PE fluorescence channel was chosen to detect $\mathrm{CD} 80^{+}$and $\mathrm{CD} 163^{+}$cells from CAR-iMACs. FACS data was collected using CytExpert, and was processed using FlowJo.

Analysis of polarization state of CAR-iMACs in vivo. To analyze the polarization state of CAR-iMACs in vivo, we first established U87MG ${ }^{\text {EGFRvIll }}$ cells derived tumor model mice using intraperitoneal injection. After 2 days of CAR-iMACs treatment, we isolated CAR-iMACs from bdominl cvity of tumor-bearing mice using dissociative buffer containing $0.02 \%$ collagenase IV (Solarbio, \#C8160), 0.01\% hyaluronidase (Meilunbio, \#37326-33-3) and 0.002\% DNAase (Meilunbio, \#9003-98-9). We then prepared two equal groups of the cells and stained either of the groups of cells using PE-CD80 antibody and PE-CD163 antibody. Then Flow cytometry was performed to detect $\mathrm{CD} 80^{+}$and $\mathrm{CD} 163^{+}$CAR-iMACs on Beckman CytoFLEX LX. The FITC channel was used to recognize EGFP-marked CAR-iMACs. PE fluorescence channel was choosed to detect $\mathrm{CD} 80^{+}$and $\mathrm{CD} 163^{+}$cells from CAR-iMACs. FACS data was collected using CytExpert (Version 2.3), and was processed using FlowJo (Version 9).

Generation of single cell GEMs and sequencing libraries. We established single cell GEMs and sequencing libraries using the methods in our previous work ${ }^{28} .10000$ cells $(90-95 \%$ viability) were captured per sample using a 10X Chromium device using 10X V2 Single Cell 3' Solution reagents (10X Genomics, Inc) at a concentration of 1000 cells/ $\mu$ l. Experiment was performed according to manual instructions. After the GEM-RT incubation, barcoded-cDNA was purified with DynaBeads cleanup mix, followed by 10 -cycles of PCR amplification $\left(98^{\circ} \mathrm{C}\right.$ for $3 \mathrm{~min}$; [ $98^{\circ} \mathrm{C}$ for $15 \mathrm{~s}, 67^{\circ} \mathrm{C}$ for $20 \mathrm{~s}, 72^{\circ} \mathrm{C}$ for $\left.1 \mathrm{~min}\right] \mathrm{x}$ $10 ; 72^{\circ} \mathrm{C}$ for $\left.1 \mathrm{~min}\right)$. The total cDNA of single-cell transcriptomes was then fragmented, double-size selected with SPRI beads (Beckman), followed by 12 cycles sample index PCR amplification $\left(98^{\circ} \mathrm{C}\right.$ for 45 
$\mathrm{s} ;\left[98^{\circ} \mathrm{C}\right.$ for $20 \mathrm{~s}, 54^{\circ} \mathrm{C}$ for $30 \mathrm{~s}, 72^{\circ} \mathrm{C}$ for $\left.1 \mathrm{~min}\right] \times 10 ; 72^{\circ} \mathrm{C}$ for $1 \mathrm{~min}$ ), subsequently another double-size selection with SPRI beads was performed before sequencing (Illumina NextSeq platform).

Public bulk RNA-sequencing data mapping and analysis. Publicly available bulk RNA-seq dataset of iPSC and macrophage cells were downloaded from two previous studies ${ }^{93,94}$. All publicly available bulk RNAseq reads were first trimmed using Trimmomatic (Version 0.36) software with the parameters "ILLUMINACLIP: TruSeq3-PE.fa:2:30:10 LEADING:3 TRAILING:3 SLIDINGWINDOW:4:15 MINLEN:36" and were further quality-filtered using the FASTX Toolkit (Version 0.0.13, http://hannonlab.cshl.edu/fastx_toolkit/) with the minimum quality score 20 and minimum percent of $80 \%$ bases that has a quality score larger than this cutoff value. The high-quality reads were mapped to the GRCh38 genome by HISAT2, a fast and sensitive spliced alignment program for mapping RNA-seq reads, with -dta parameter. PCR duplicate reads were removed using Picard tools (v2.18.2) and only uniquely mapped reads were kept for further analysis. The expression levels of genes were calculated by StringTie (Version v1.3.4d, with -e -B -G parameters) using Release 28 (GRCh38.p12) gene annotations downloaded from GENCODE data portal. To obtain reliable and cross-sample comparable expression abundance estimation for each gene, reads mapped to the reference genome were counted as TPM (Transcripts Per Million reads) based on their genome locations. We used "prcomp" and pheatmap function with default parameters for Principal Component Analysis (PCA) and hierarchical clustering, respectively. We used high confident genes characterizing the $M 1$ and $M 2$ states (with the maximum of TPM values among different samples larger than 1) for PCA and hierarchical clustering analysis.

Single cell RNA-sequencing data analysis. The "cellrange count" program, a subcommand included in 10X single-cell gene expression analysis pipeline (https://support.10xgenomics.com/single-cell-geneexpression/), was used to produce gene-cell barcode expression matrix. The single-cell gene expression matrix further was analyzed with Seurat v3.2.1 (https://satijalab.org/seurat/). To guarantee those quality of genes and cells used for downstream analysis, we excluded the genes with expressed cell number smaller than 3 and those cells with expressed genes smaller than 200 or larger than 30000 . We also filtered the cells with the expression percentages of mitochondrial genes larger than 0.2 . We then adopted 10 Principal Components (PCs) for tSNE and clustering analysis with the cluster resolution of 0.25 .

The known cell identities (types) of each cell cluster of gene expression data were further predicted by an entropy-based predictor with default parameters (http://scibet.cancer-pku.cn/) (SciBet v1.0). To perform a comprehensive annotation of well-known cell types, weusedd an atlas database of cell types during human fetal liver haematopoiesi ${ }^{95}$, and integrate them with 30 major human cell type databases from 42 single cell RNA-seq datasets ${ }^{96}$. To perform Principal Component Analysis (PCA) and hierarchical clustering analysis on bulk RNA-seq data, we have grouped and summed up the normalized expression levels of our single-cell gene expression data to produce the synthetic bulk RNA-seq dataset. We used "prcomp" and pheatmap function with default parameters for PCA and hierarchical clustering, respectively. All the bioinformatic data analyses and resulting visualization were performed in $\mathrm{R}$ software (4.0.2)/Bioconductor (v3.13) utilizing custom R scripts. 


\section{References}

1. June, C. H., O'Connor, R. S., Kawalekar, O. U., Ghassemi, S. \& Milone, M. C. CAR T cell immunotherapy for human cancer. Science 359, 1361-1365, doi:10.1126/science.aar6711 (2018).

2. Wang, Z., Wu, Z., Liu, Y. \& Han, W. New development in CAR-T cell therapy. Journal of hematology \& oncology 10, 53, doi:10.1186/s13045-017-0423-1 (2017).

3. Bunse, M., Pfeilschifter, J., Bluhm, J., Zschummel, M., Joedicke, J. J., Wirges, A,... Höpken, U. E. CXCR5 CAR-T cells simultaneously target B cell non-Hodgkin's lymphoma and tumor-supportive follicular T helper cells. Nature communication 12, 240, doi:10.1038/s41467-020-20488-3 (2021).

4. Gomes-Silva, D., Srinivasan, M., Sharma, S., Lee, C. M., Wagner, D. L., Davis, T. H.,.. . Mamonkin, M. CD7-edited T cells expressing a CD7-specific CAR for the therapy of T-cell malignancies. Blood 130, 285-296, doi:10.1182/blood-2017-01-761320 (2017).

5. June, C. H. \& Sadelain, M. Chimeric Antigen Receptor Therapy. The New England journal of medicine 379, 64-73, doi:10.1056/NEJMra1706169 (2018).

6. Sadelain, M., Rivière, I. \& Riddell, S. Therapeutic T cell engineering. Nature 545, 423-431, doi:10.1038/nature22395 (2017).

7. Schuster, S. J., Svoboda, J., Chong, E. A., Nasta, S. D., Mato, A. R., Anak, Ö.,.. . June, C. H. Chimeric Antigen Receptor T Cells in Refractory B-Cell Lymphomas. The New England journal of medicine 377, 2545-2554, doi:10.1056/NEJMoa1708566 (2017).

8. Akhavan, D., Alizadeh, D., Wang, D., Weist, M. R., Shepphird, J. K. \& Brown, C. E. CAR T cells for brain tumors: Lessons learned and road ahead. Immunological reviews 290, 60-84, doi:10.1111/imr.12773 (2019).

9. Chuntova, P., Downey, K. M., Hegde, B., Almeida, N. D. \& Okada, H. Genetically Engineered T-Cells for Malignant Glioma: Overcoming the Barriers to Effective Immunotherapy. Frontiers in immunology 9 , 3062, doi:10.3389/fimmu.2018.03062 (2018).

10. D'Aloia, M. M., Zizzari, I. G., Sacchetti, B., Pierelli, L. \& Alimandi, M. CAR-T cells: the long and winding road to solid tumors. Cell death \& disease 9, 282, doi:10.1038/s41419-018-0278-6 (2018).

11. Ma, L. \& Dichwalkar, T. Enhanced CAR-T cell activity against solid tumors by vaccine boosting through the chimeric receptor. 365, 162-168, doi:10.1126/science.aav8692 (2019).

12. Lynn, R. C., Weber, E. W., Sotillo, E., Gennert, D., Xu, P., Good, Z.,.. . Mackall, C. L. c-Jun overexpression in CAR T cells induces exhaustion resistance. Nature 576, 293-300, doi:10.1038/s41586-019-1805-Z (2019).

13. Wherry, E. J. \& Kurachi, M. Molecular and cellular insights into T cell exhaustion. Nature reviews. Immunology 15, 486-499, doi:10.1038/nri3862 (2015).

14. Srivastava, S., Furlan, S. N., Jaeger-Ruckstuhl, C. A., Sarvothama, M., Berger, C., Smythe, K. S.,.. . Riddell, S. R. Immunogenic Chemotherapy Enhances Recruitment of CAR-T Cells to Lung Tumors and Improves Antitumor Efficacy when Combined with Checkpoint Blockade. Cancer cell 39, 193208.e110, doi:10.1016/j.ccell.2020.11.005 (2021). 
15. Singh, A. K. \& McGuirk, J. P. CAR T cells: continuation in a revolution of immunotherapy. The Lancet. Oncology 21, e168-e178, doi:10.1016/s1470-2045(19)30823-x (2020).

16. Adachi, K., Kano, Y., Nagai, T., Okuyama, N., Sakoda, Y. \& Tamada, K. IL-7 and CCL19 expression in CAR-T cells improves immune cell infiltration and CAR-T cell survival in the tumor. Nature biotechnology 36, 346-351, doi:10.1038/nbt.4086 (2018).

17. Cantor, J. M., Rose, D. M., Slepak, M. \& Ginsberg, M. H. Fine-tuning Tumor Immunity with Integrin Trans-regulation. Cancer immunology research 3, 661-667, doi:10.1158/2326-6066.cir-13-0226 (2015).

18. Nguyen, N. T.,Huang, K., Zeng, H., Jing, J., Wang, R., Fang, S., Chen, J.,... Zhou, Y. Nano-optogenetic engineering of CAR T cells for precision immunotherapy with enhanced safety. Nature nanotechnology 16, 1424-1434, doi:10.1038/s41565-021-00982-5 (2021).

19. Brentjens, R. J., Hackett, C. S., Brentjens, R. J. Engineering strategies to overcome the current roadblocks in CAR T cell therapy. Nature reviews. Clinical oncology, doi:10.1038/s41571-019-0297-y (2019).

20. Martinez, M. \& Moon, E. K. CAR T Cells for Solid Tumors: New Strategies for Finding, Infiltrating, and Surviving in the Tumor Microenvironment. Frontiers in immunology 10, 128, doi:10.3389/fimmu.2019.00128 (2019).

21. Balkwill, F. \& Mantovani, A. Inflammation and cancer: back to Virchow? Lancet (London, England) 357, 539-545, doi:10.1016/s0140-6736(00)04046-0 (2001).

22. Guilliams, M., Mildner, A. \& Yona, S. Developmental and Functional Heterogeneity of Monocytes. Immunity 49, 595-613, doi:10.1016/j.immuni.2018.10.005 (2018).

23. Bonnardel, J. \& Guilliams, M. Developmental control of macrophage function. Current opinion in immunology 50, 64-74, doi:10.1016/j.coi.2017.12.001 (2018).

24. Tacke, F. Targeting hepatic macrophages to treat liver diseases. Journal of hepatology $66,1300-$ 1312, doi:10.1016/j.jhep.2017.02.026 (2017).

25. Cassetta, L. \& Pollard, J. W. Targeting macrophages: therapeutic approaches in cancer. Nature reviews. Drug discovery 17, 887-904, doi:10.1038/nrd.2018.169 (2018).

26. Davies, L. C. \& Taylor, P. R. Tissue-resident macrophages: then and now. Immunology 144, 541-548, doi:10.1111/imm.12451 (2015).

27. Mantovani, A., Marchesi, F., Malesci, A., Laghi, L. \& Allavena, P. Tumour-associated macrophages as treatment targets in oncology. Nature reviews. Clinical oncology 14, 399-416, doi:10.1038/nrclinonc.2016.217 (2017).

28. Vonderheide, R. H. CD40 Agonist Antibodies in Cancer Immunotherapy. Annual review of medicine 71, 47-58, doi:10.1146/annurev-med-062518-045435 (2020).

29. Zhang, L., Tian, L., Dai, X., Yu, H., Wang, J., Lei, A.,.. . Zhang, J. Pluripotent stem cell-derived CARmacrophage cells with antigen-dependent anti-cancer cell functions. Journal of hematology \& oncology 13, 153, doi:10.1186/s13045-020-00983-2 (2020). 
30. Anderson, N. R., Minutolo, N. G., Gill, S. \& Klichinsky, M. Macrophage-Based Approaches for Cancer Immunotherapy. Cancer research 81, 1201-1208, doi:10.1158/0008-5472.can-20-2990 (2021).

31. Klichinsky, M., Ruella, M., Shestova, O., Lu, X. M., Best, A., Zeeman, M.,... Gill, S. Human chimeric antigen receptor macrophages for cancer immunotherapy. Nature biotechnology 38, 947-953, doi:10.1038/s41587-020-0462-y (2020).

32. Cortez-Retamozo, V., Etzrodt, M., Newton, A., Rauch, P. J., Chudnovskiy, A., Berger, C.,.. . Pittet, M. J. Origins of tumor-associated macrophages and neutrophils. Proceedings of the National Academy of Sciences of the United States of America 109, 2491-2496, doi:10.1073/pnas.1113744109 (2012).

33. Hui, L. \& Chen, Y. Tumor microenvironment: Sanctuary of the devil. Cancer letters 368, 7-13, doi:10.1016/j.canlet.2015.07.039 (2015).

34. Hinshaw, D. C. \& Shevde, L. A. The Tumor Microenvironment Innately Modulates Cancer Progression. Cancer research 79, 4557-4566, doi:10.1158/0008-5472.can-18-3962 (2019).

35. Mantovani, A., Sozzani, S., Locati, M., Allavena, P. \& Sica, A. Macrophage polarization: tumorassociated macrophages as a paradigm for polarized M2 mononuclear phagocytes. Trends in immunology 23, 549-555, doi:10.1016/s1471-4906(02)02302-5 (2002).

36. Qian, B. Z. \& Pollard, J. W. Macrophage diversity enhances tumor progression and metastasis. Cell 141, 39-51, doi:10.1016/j.cell.2010.03.014 (2010).

37. Gambardella, V., Castillo, J., Tarazona, N., Gimeno-Valiente, F., Martínez-Ciarpaglini, C., CabezaSegura, M.,... Fleitas, T. The role of tumor-associated macrophages in gastric cancer development and their potential as a therapeutic target. Cancer treatment reviews 86,102015 , doi:10.1016/j.ctrv.2020.102015 (2020).

38. Chen, Y., Zhang, S., Wang, Q. \& Zhang, X. Tumor-recruited M2 macrophages promote gastric and breast cancer metastasis via M2 macrophage-secreted CHI3L1 protein. Journal of hematology \& oncology 10, 36, doi:10.1186/s13045-017-0408-0 (2017).

39. DeNardo, D. G. \& Ruffell, B. Macrophages as regulators of tumour immunity and immunotherapy. Nature reviews. Immunology 19, 369-382, doi:10.1038/s41577-019-0127-6 (2019).

40. Nowak, M. \& Klink, M. The Role of Tumor-Associated Macrophages in the Progression and Chemoresistance of Ovarian Cancer. cells 9, doi:10.3390/cells9051299 (2020).

41. Purdon, T., Dong, X., Zhu, L.,... Ma, X. Tumor derived UBR5 promotes ovarian cancer growth and metastasis through inducing immunosuppressive macrophages. Nature communication 11,6298 , doi:10.1038/s41467-020-20140-0 (2020).

42. van Beijnum, J. R., Melis, M. H. M., van Burgsteden, J., Muraro, M, J.,... Bergman,A. M. Androgen receptor signalling in macrophages promotes TREM-1-mediated prostate cancer cell line migration and invasion. Nature communication 11, 4498, doi:10.1038/s41467-020-18313-y (2020).

43. Huang, R., Wang, S., Wang, N., Zheng, Y., Zhou, J., Yang, B.,... Xiang, S. CCL5 derived from tumorassociated macrophages promotes prostate cancer stem cells and metastasis via activating $\beta$ catenin/STAT3 signaling. Cell death \& disease, doi:10.1038/s41419-020-2435-y (2020). 
44. Salvagno, C., Ciampricotti, M., Tuit, S., Hau, C. S., van Weverwijk, A. \& Coffelt, S. B. Therapeutic targeting of macrophages enhances chemotherapy efficacy by unleashing type I interferon response. Nature cell biology 21, 511-521, doi:10.1038/s41556-019-0298-1 (2019).

45. Wiehagen, K. R., Girgis, N. M., Yamada, D. H., Smith, A. A., Chan, S. R., Grewal, I. S.,.. . Verona, R. I. Combination of CD40 Agonism and CSF-1R Blockade Reconditions Tumor-Associated Macrophages and Drives Potent Antitumor Immunity. Cancer immunology research 5, 1109-1121, doi:10.1158/2326-6066.cir-17-0258 (2017).

46. Kang, M., Lee, S. H., Kwon, M., Byun, J., Kim, D., Kim, C.,.. . Kim, B. S. Nanocomplex-Mediated In Vivo Programming to Chimeric Antigen Receptor-M1 Macrophages for Cancer Therapy. Advanced materials 33, e2103258, doi:10.1002/adma.202103258 (2021).

47. Ries, C. H., Cannarile, M. A., Hoves, S., Benz, J., Wartha, K., Runza, V.,.. . Rüttinger, D. Targeting tumorassociated macrophages with anti-CSF-1R antibody reveals a strategy for cancer therapy. Cancer cell 25, 846-859, doi:10.1016/j.ccr.2014.05.016 (2014).

48. Klemm, F., Handgraaf, S. M., de Groot, M.,... Joyce, J. A. Dynamic changes in glioma macrophage populations after radiotherapy reveal CSF-1R inhibition as a strategy to overcome resistance. Science translational medicine 12, doi:10.1126/scitranslmed.aaw7843 (2020).

49. Morrissey, M. A. Williamson, A. P., Steinbach, A. M., Roberts, E. W., Kern, N., Headley, M. B. \& Vale, R. D. Chimeric antigen receptors that trigger phagocytosis. Elife 7, doi:10.7554/eLife.36688 (2018).

50. Sunseri, N., O'Brien, M., Bhardwaj, N. \& Landau, N. R. Human immunodeficiency virus type 1 modified to package Simian immunodeficiency virus Vpx efficiently infects macrophages and dendritic cells. Journal of virology 85, 6263-6274, doi:10.1128/jvi.00346-11 (2011).

51. Sloas, C., Gill, S. \& Klichinsky, M. Engineered CAR-Macrophages as Adoptive Immunotherapies for Solid Tumors. Frontiers in immunology 12, 783305, doi:10.3389/fimmu.2021.783305 (2021).

52. De Palma, M., Mazzieri, R., Politi, L. S., Pucci, F., Zonari, E., Sitia, G.,.. . Naldini, L. Tumor-targeted interferon-alpha delivery by Tie2-expressing monocytes inhibits tumor growth and metastasis. Cancer cell 14, 299-311, doi:10.1016/j.ccr.2008.09.004 (2008).

53. Zhang, W., Liu, L., Su, H., Liu, Q., Shen, J., Dai, H.,.. . Shen, P. Chimeric antigen receptor macrophage therapy for breast tumours mediated by targeting the tumour extracellular matrix. British journal of cancer 121, 837-845, doi:10.1038/s41416-019-0578-3 (2019).

54. Brempelis, K. J., Cowan, C. M., Kreuser, S. A., Labadie, K. P., Prieskorn, B. M., Lieberman, N. A. P.,.. . Crane, C. A. Genetically engineered macrophages persist in solid tumors and locally deliver therapeutic proteins to activate immune responses. Journal for immunotherapy of cancer $\mathbf{8}$, doi:10.1136/jitc-2020-001356 (2020).

55. Gardell, J. L., Matsumoto, L. R., Chinn, H., DeGolier, K. R., Kreuser, S. A., Prieskorn, B.,.. . Crane, C. A. Human macrophages engineered to secrete a bispecific $T$ cell engager support antigen-dependent $T$ cell responses to glioblastoma. Journal for immunotherapy of cancer $\mathbf{8}$, doi:10.1136/jitc-2020001202 (2020). 
56. Takata, K., Kozaki, T., Lee, C. Z. W., Thion, M. S., Otsuka, M., Lim, S.,.. . Ginhoux, F. Induced-PluripotentStem-Cell-Derived Primitive Macrophages Provide a Platform for Modeling Tissue-Resident Macrophage Differentiation and Function. Immunity 47, 183-198.e186, doi:10.1016/j.immuni.2017.06.017 (2017).

57. Zhang, H. \& Reilly, M. P. Human Induced Pluripotent Stem Cell-Derived Macrophages for Unraveling Human Macrophage Biology. Arteriosclerosis, thrombosis, and vascular biology 37, 2000-2006, doi:10.1161/atvbaha.117.309195 (2017).

58. Lachmann, N., Ackermann, M., Frenzel, E., Liebhaber, S., Brennig, S., Happle, C.,.. . Moritz, T. Largescale hematopoietic differentiation of human induced pluripotent stem cells provides granulocytes or macrophages for cell replacement therapies. Stem cell reports 4, 282-296, doi:10.1016/j.stemcr.2015.01.005 (2015).

59. Kawai, T. \& Akira, S. Toll-like receptors and their crosstalk with other innate receptors in infection and immunity. Immunity 34,637-650, doi:10.1016/j.immuni.2011.05.006 (2011).

60. Blasius, A. L. \& Beutler, B. Intracellular toll-like receptors. Immunity 32, 305-315, doi:10.1016/j.immuni.2010.03.012 (2010).

61. Akira, S., Uematsu, S. \& Takeuchi, O. Pathogen recognition and innate immunity. Cell 124, 783-801, doi:10.1016/j.cell.2006.02.015 (2006).

62. Medzhitov, R., Preston-Hurlburt, P. \& Janeway, C. A., Jr. A human homologue of the Drosophila Toll protein signals activation of adaptive immunity. Nature 388, 394-397, doi:10.1038/41131 (1997).

63. Lancaster, G. I., Langley, K. G., Berglund, N. A., Kammoun, H. L., Reibe, S., Estevez, E.,.. . Febbraio, M. A. Evidence that TLR4 Is Not a Receptor for Saturated Fatty Acids but Mediates Lipid-Induced Inflammation by Reprogramming Macrophage Metabolism. Cell metabolism 27, 1096-1110.e1095, doi:10.1016/j.cmet.2018.03.014 (2018).

64. Fan, W., Morinaga, H., Kim, J. J., Bae, E., Spann, N. J., Heinz, S.,.. . Olefsky, J. M. FoxO1 regulates TIr4 inflammatory pathway signalling in macrophages. The EMBO journal 29, 4223-4236, doi:10.1038/emboj.2010.268 (2010).

65. Fitzgerald, K. A. \& Kagan, J. C. Toll-like Receptors and the Control of Immunity. Cell 180, 1044-1066, doi:10.1016/j.cell.2020.02.041 (2020).

66. Kawai, T. \& Akira, S. The role of pattern-recognition receptors in innate immunity: update on Toll-like receptors. Nature immunology 11, 373-384, doi:10.1038/ni.1863 (2010).

67. Kondo, T., Kawai, T. \& Akira, S. Dissecting negative regulation of Toll-like receptor signaling. Trends in immunology 33, 449-458, doi:10.1016/j.it.2012.05.002 (2012).

68. Schappe, M. S., Szteyn, K., Stremska, M. E., Mendu, S. K., Downs, T. K., Seegren, P. V.,.. . Desai, B. N. Chanzyme TRPM7 Mediates the Ca(2+) Influx Essential for Lipopolysaccharide-Induced Toll-Like Receptor 4 Endocytosis and Macrophage Activation. Immunity 48, 59-74.e55, doi:10.1016/j.immuni.2017.11.026 (2018).

69. Kagan, J. C., Su, T., Horng, T., Chow, A., Akira, S. \& Medzhitov, R. TRAM couples endocytosis of Tolllike receptor 4 to the induction of interferon-beta. Nature immunology 9, 361-368, 
doi:10.1038/ni1569 (2008).

70. Yamamoto, M., Sato, S., Hemmi, H., Hoshino, K., Kaisho, T., Sanjo, H.,.. . Akira, S. Role of adaptor TRIF in the MyD88-independent toll-like receptor signaling pathway. Science (New York, N.Y.) 301, 640643, doi:10.1126/science.1087262 (2003).

71. Wermuth, P. J. \& Jimenez, S. A. Gadolinium compounds signaling through TLR4 and TLR7 in normal human macrophages: establishment of a proinflammatory phenotype and implications for the pathogenesis of nephrogenic systemic fibrosis. Journal of immunology (Baltimore, Md.: 1950) 189, 318-327, doi:10.4049/jimmunol.1103099 (2012).

72. Wong, V., Scott-Browne, J. P., Togher, S., Hoffmann, A.,... Trifari, S. Exhaustion-associated regulatory regions in CD8(+) tumor-infiltrating T cells. Proceedings of the National Academy of Sciences of the United States of America114, E2776-e2785, doi:10.1073/pnas.1620498114 (2017).

73. Martínez, V. G., Rubio, C., Martínez-Fernández, M., Segovia, C., López-Calderón, F., Garín, M. I.,.. . Dueñas, M. BMP4 Induces M2 Macrophage Polarization and Favors Tumor Progression in Bladder Cancer. Clinical cancer research: an official journal of the American Association for Cancer Research 23, 7388-7399, doi:10.1158/1078-0432.ccr-17-1004 (2017).

74. Yu, T., Gan, S., Zhu, Q., Dai, D., Li, N., Wang, H.,.. . Peng, C. Modulation of M2 macrophage polarization by the crosstalk between Stat 6 and Trim24. Nature communication 10, 4353, doi:10.1038/s41467019-12384-2 (2019).

75. Lu, C. S., Shiau, A. L., Su, B. H., Hsu, T. S., Wang, C. T., Su, Y. C.,.. . Shieh, G. S. Oct4 promotes M2 macrophage polarization through upregulation of macrophage colony-stimulating factor in lung cancer. Journal of hematology \& oncology 13, 62, doi:10.1186/s13045-020-00887-1 (2020).

76. Moynagh, P. N. TLR signalling and activation of IRFs: revisiting old friends from the NF-kappaB pathway. Trends in immunology 26, 469-476, doi:10.1016/j.it.2005.06.009 (2005).

77. Jaroonwitchawan, T., Visitchanakun, P., Dang, P. C., Ritprajak, P., Palaga, T. \& Leelahavanichkul, A. Dysregulation of Lipid Metabolism in Macrophages Is Responsible for Severe Endotoxin Tolerance in FcgRIIB-Deficient Lupus Mice. Frontiers in immunology 11, 959, doi:10.3389/fimmu.2020.00959 (2020).

78. Novakovic, B., Habibi, E., Wang, S. Y., Arts, R. J. W., Davar, R., Megchelenbrink, W.,.. . Stunnenberg, H. G. $\beta$-Glucan Reverses the Epigenetic State of LPS-Induced Immunological Tolerance. Cell 167, 13541368.e1314, doi:10.1016/j.cell.2016.09.034 (2016).

79. Butcher, S. K., O'Carroll, C. E., Wells, C. A. \& Carmody, R. J. Toll-Like Receptors Drive Specific Patterns of Tolerance and Training on Restimulation of Macrophages. Frontiers in immunology 9, 933, doi:10.3389/fimmu.2018.00933 (2018).

80. Carmody, R. J., Ruan, Q., Palmer, S., Hilliard, B. \& Chen, Y. H. Negative regulation of toll-like receptor signaling by NF-kappaB p50 ubiquitination blockade. Science (New York, N.Y.) 317, 675-678, doi:10.1126/science.1142953 (2007).

81. Jung, J., Shibata, M., Aksoylar, H. I., Lei, J.,... Horng, T. Glycerol phosphate shuttle enzyme GPD2 regulates macrophage inflammatory responses. Nature immunology 20, 1186-1195, 
doi:10.1038/s41590-019-0453-7 (2019).

82. Franklin, R. A., Liao, W., Sarkar, A., Kim, M. V., Bivona, M. R., Liu, K.,.. . Li, M. O. The cellular and molecular origin of tumor-associated macrophages. Science (New York, N.Y.) 344, 921-925, doi:10.1126/science.1252510 (2014).

83. Chen, Y., Yu, Z., Tan, X., Jiang, H., Xu, Z., Fang, Y.,.. . Tu, J. CAR-macrophage: A new immunotherapy candidate against solid tumors. Biomedicine \& pharmacotherapy = Biomedecine \& pharmacotherapie 139, 111605, doi:10.1016/j.biopha.2021.111605 (2021).

84. Hong, M., Clubb, J. D. \& Chen, Y. Y. Engineering CAR-T Cells for Next-Generation Cancer Therapy. Cancer cell 38, 473-488, doi:10.1016/j.ccell.2020.07.005 (2020).

85. Sadelain, M., Brentjens, R. \& Rivière, I. The promise and potential pitfalls of chimeric antigen receptors. Current opinion in immunology 21, 215-223, doi:10.1016/j.coi.2009.02.009 (2009).

86. Feins, S., Kong, W., Williams, E. F., Milone, M. C. \& Fraietta, J. A. An introduction to chimeric antigen receptor (CAR) T-cell immunotherapy for human cancer. American journal of hematology 94, S3-s9, doi:10.1002/ajh.25418 (2019).

87. Maldini, C. R., Claiborne, D. T., Okawa, K., Chen, T., Dopkin, D. L., Shan, X.,.. . Trifonova, R. T. Dual CD4based CAR T cells with distinct costimulatory domains mitigate HIV pathogenesis in vivo. Nature medicine 26, 1776-1787, doi:10.1038/s41591-020-1039-5 (2020).

88. Kawalekar, O. U., O'Connor, R. S., Fraietta, J. A., Guo, L., McGettigan, S. E., Posey, A. D., Jr.,.. . June, C. H. Distinct Signaling of Coreceptors Regulates Specific Metabolism Pathways and Impacts Memory Development in CAR T Cells. Immunity 44, 380-390, doi:10.1016/j.immuni.2016.01.021 (2016).

89. Drent, E., Poels, R., Ruiter, R., van de Donk, N., Zweegman, S., Yuan, H.,.. . Themeli, M. Combined CD28 and 4-1BB Costimulation Potentiates Affinity-tuned Chimeric Antigen Receptor-engineered T Cells. Clinical cancer research: an official journal of the American Association for Cancer Research 25, 4014-4025, doi:10.1158/1078-0432.ccr-18-2559 (2019).

90. Cappell, K. M. \& Kochenderfer, J. N. A comparison of chimeric antigen receptors containing CD28 versus 4-1BB costimulatory domains. Nature revieew. clinical oncology $18,715-727$, doi:10.1038/s41571-021-00530-z (2021).

91. Haynes, N. M., Trapani, J. A., Teng, M. W., Jackson, J. T., Cerruti, L., Jane, S. M.,.. . Darcy, P. K. Singlechain antigen recognition receptors that costimulate potent rejection of established experimental tumors. Blood 100, 3155-3163, doi:10.1182/blood-2002-04-1041 (2002).

92. Maher, J., Brentjens, R. J., Gunset, G., Rivière, I. \& Sadelain, M. Human T-lymphocyte cytotoxicity and proliferation directed by a single chimeric TCRzeta /CD28 receptor. Nature biotechnology $20,70-75$, doi:10.1038/nbt0102-70 (2002).

93. Zhang, H., Xue, C., Shah, R., Bermingham, K., Hinkle, C. C., Li, W.,.. Reilly, M. P. Functional analysis and transcriptomic profiling of iPSC-derived macrophages and their application in modeling Mendelian disease. Circulation research 117, 17-28, doi:10.1161/circresaha.117.305860 (2015).

94. Douvaras, P., Sun, B., Wang, M., Kruglikov, I., Lallos, G., Zimmer, M.,.. . Fossati, V. Directed Differentiation of Human Pluripotent Stem Cells to Microglia. Stem cell reports 8, 1516-1524, 
doi:10.1016/j.stemcr.2017.04.023 (2017).

95. Popescu, D. M., Botting, R. A., Stephenson, E., Green, K., Webb, S., Jardine, L.,.. . Haniffa, M. Decoding human fetal liver haematopoiesis. Nature 574, 365-371, doi:10.1038/s41586-019-1652-y (2019).

96. Liu, B., Li, C., Li, Z., Wang, D., Ren, X. \& Zhang, Z. An entropy-based metric for assessing the purity of single cell populations. Nature communication 11, 3155, doi:10.1038/s41467-020-16904-3 (2020).

\section{Figures}



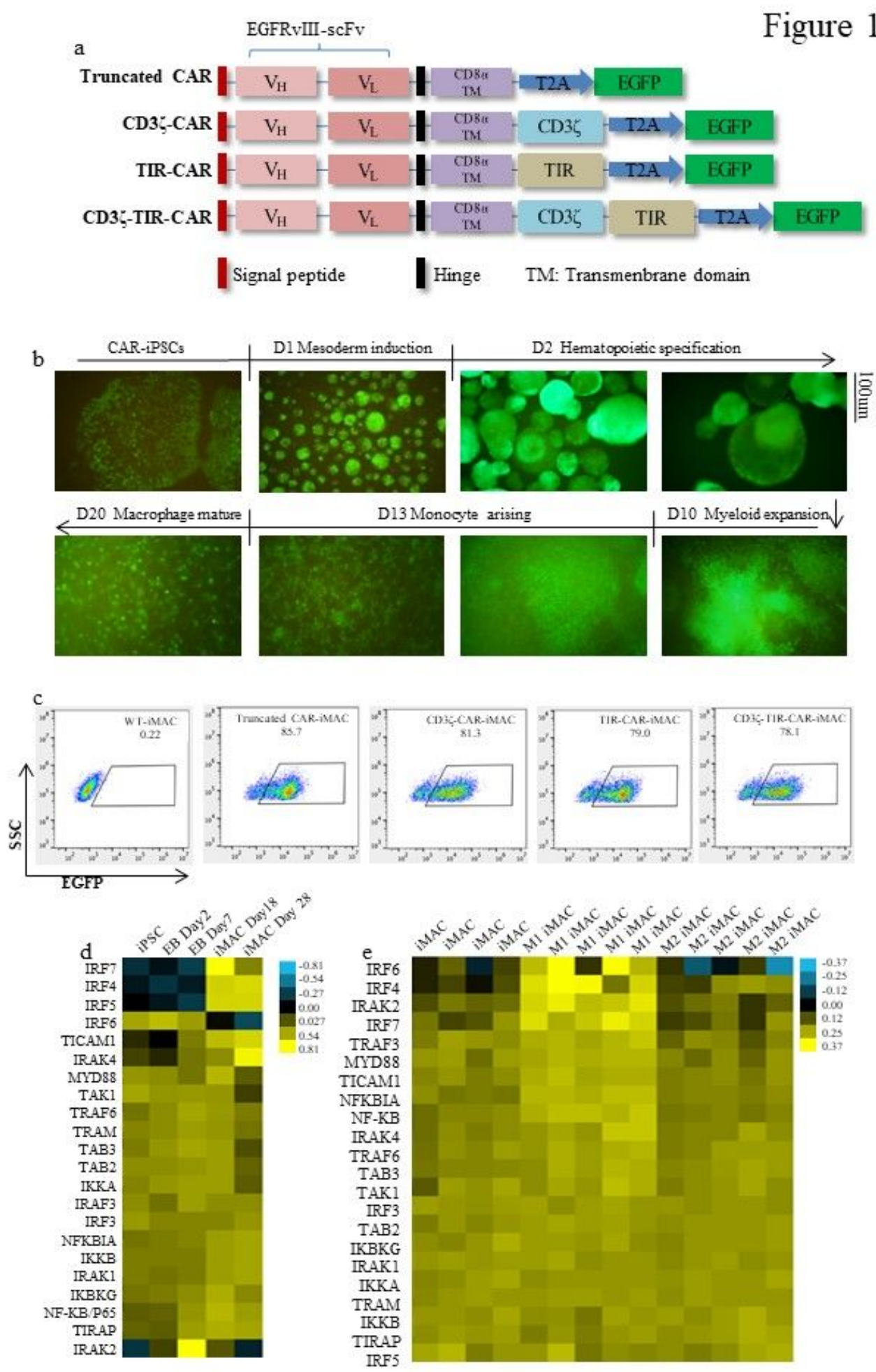

\section{Figure 1}

Designs of CAR constructs and production of CAR-iMAC cells. a, Schematic diagram of CAR constructs. The CARs mainly comprise an extracellular signal peptide, a single-chain fragment variable (scFv) recognizing EGFRvIll, a transmembrane (TM) domain from CD8a, and either without an intracellular

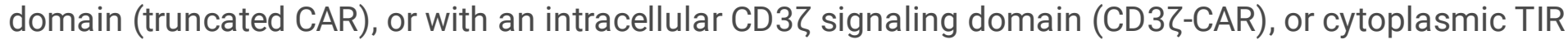

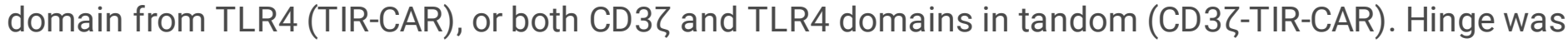


added between scFv and CD8a transmembrane domains to endow the CARs with flexibility to target antigens. All the CAR transgenes were linked to EGFP expressing sequence via a T2A element. $\mathbf{b}$, Overview of the differentiation process of CAR-iMACs from the CAR-expressing iPSCs. EGFP-labeled CARiPSCs were differentiated into CAR-iMACs by mimicking the in vivo process of mesoderm induction, hematopoietic stem cell specification and myeloid cell production. c, Flow cytometry analysis was performed to determine the transduction efficiency of CAR expression in iMACs through counting the percentage of EGFP-expressing cells. $\mathbf{d}$, A heat map from RNA-sequencing shows dynamic changes of the expression levels of TLR4 signaling related modulator genes along with the differentiation process from iPSCs to mature iMACs. The samples were taken from undifferentiated iPSC population, EBs of day 2 and day 7, and iMACs at day 18 and day 28. e, Comparison of expression level of TLR4 pathway-related adaptor and signal transduction genes in the in vitro cultured unstimulated iMACs, LPS/IFN-Y-polarized M1 iMACs and IL-4/IL-10-polarized M2 iMACs $\square$ 


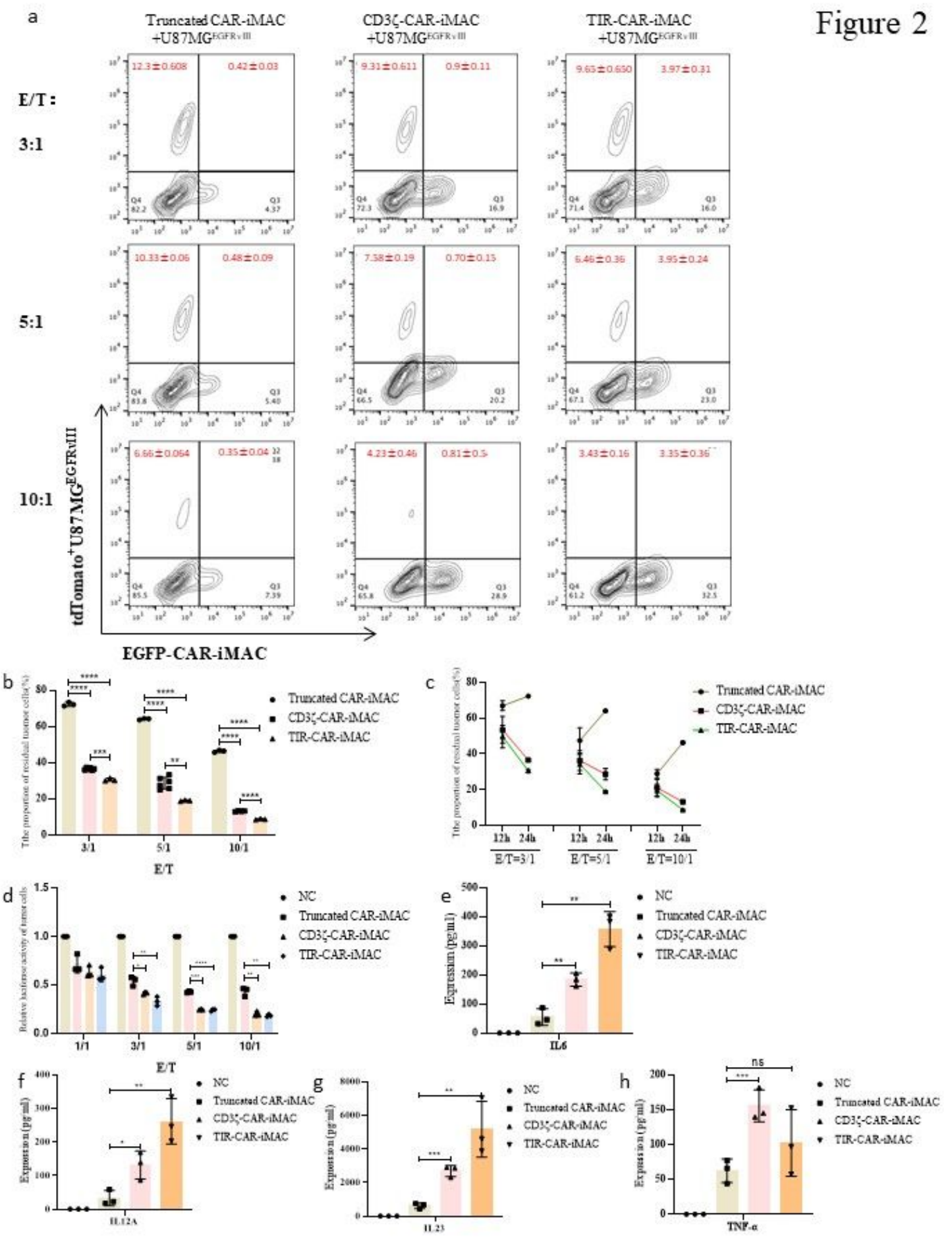

\section{Figure 2}

Both CD3Z and TIR domains conferred antigen-dependent cytotoxic activity of CAR-iMACs against U87MG ${ }^{\text {EGFRvIll }}$ cells in vitro. a, Flow cytometry analyses showing the proportion of EGFP-marked CARiMACs and tdTomato-expressed U87MGEGFRvll cells after co-incubation for 24 hours. The cells with no fluorescence protein expressed were used as blank controls. Truncated CAR-iMACs, CD3Z-CAR-iMACs and TIR-CAR-iMACs were cultured with U87MG ${ }^{\text {EGFRvill }}$ cells in the condition of effect/target ratios (E/T) of 3/1, 
$5 / 1$ and $10 / 1$, respectively. b. Statistics showing the proportion of residual tdTomato-expressed U87MG ${ }^{E G F R v I l l}$ cells from a. c, Progressive cytotoxic activity shown by the proportion of residual U87MG EGFRvill cells from b (24 hours incubation) and Supplementary Fig. $2 b$ ( 12 hours incubation). d, Statistics of bioluminescence from residual FFluc ${ }^{+} U 87 M^{\text {EGFRvIll }}$ cells after incubating with the three types of CAR-iMACs for 24 hours. E/T ratios were set as 1/1,3/1, 5/1 and 10/1. The experimental groups without CAR-iMACs treatment were designed as negative controls (NC). eh, After 24 hours co-incubation

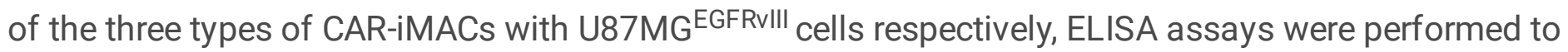
detect production of IL6 (e), IL12A (f), IL23(g), and TNF-a(h) in the media of the individual experimental group. All the data was shown by Graphpad Prism 8.2.1. $n=3$. Error bar: SEM. Significance was calculated

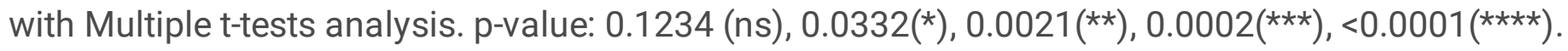


Figure 3
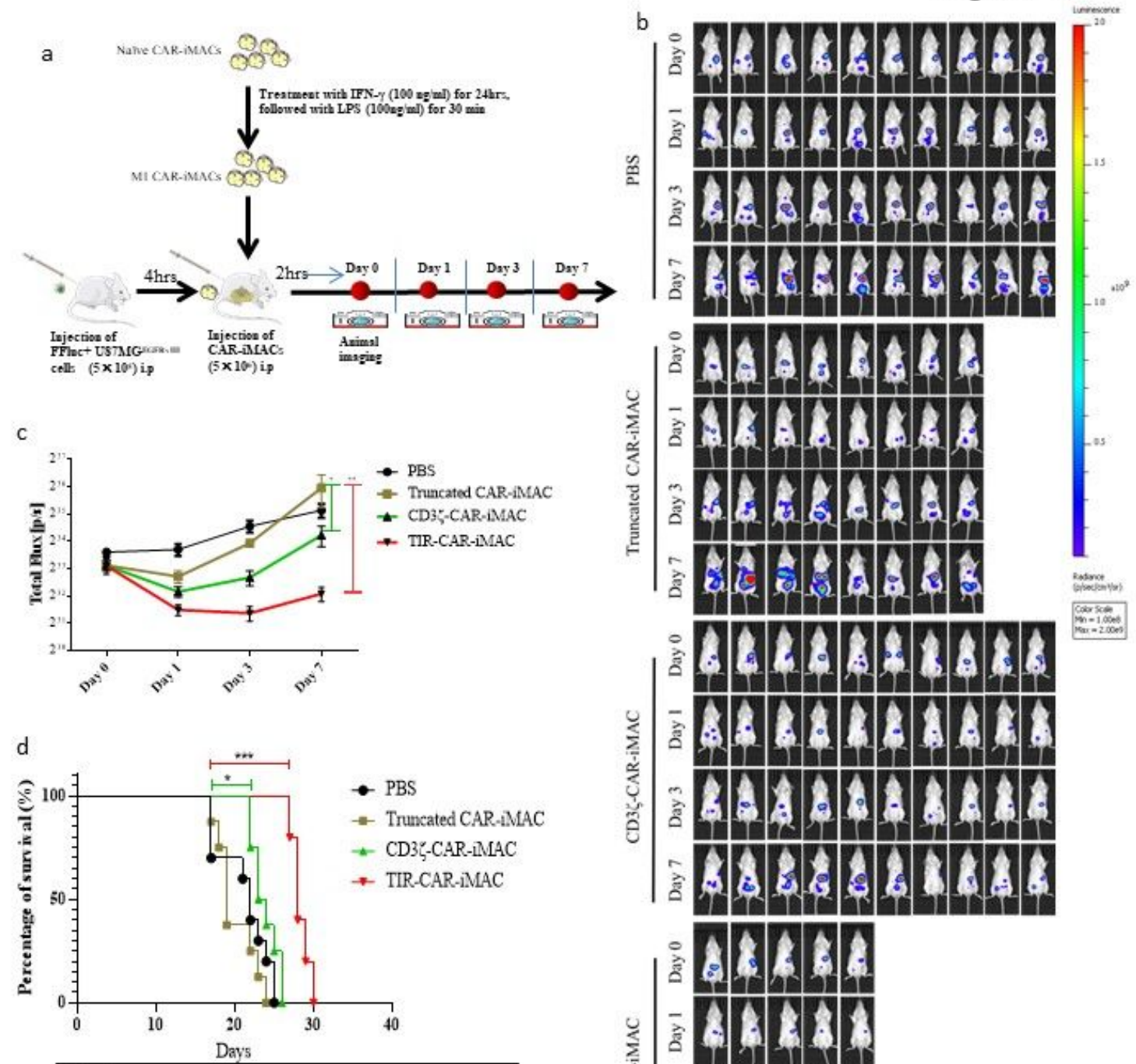

P-value:

Truncated-CAR-iMACVS PBS :0.281

CD3C-CAR-IMACVS PBS : 0.0937

TIR-CAR-iMACVS PBS : 0.0006

CD3C-CAR-iMAC VS Truncate-CAR-iMAC : 0.0115

TIR-CAR-iMAC VS Truncate-CAR-iMAC : 0.0007

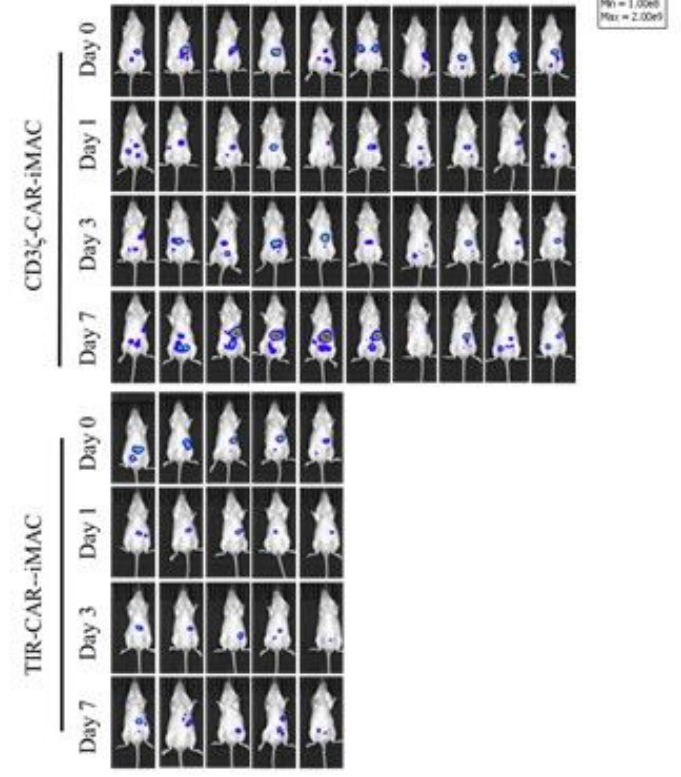

\section{Figure 3}

TIR-CAR-iMACs showed more robust anti-tumor activity than CD3Z-CAR-iMACs in vivo. a, The sketch map of the anti-tumor assay of CAR-iMACs. The truncated CAR-iMACs, CD3Z-CAR-iMACs and TIR-CAR-iMACs were pretreated with IFN- $y(100 \mathrm{ng} / \mathrm{ml})$ for 24 hours, as well as LPS $(100 \mathrm{ng} / \mathrm{ml})$ for $30 \mathrm{~min}$, and then labeled with the DiR dye, followed by intraperitoneal injection of the above cells into NSG mice innoculated with FFluc ${ }^{+} U 87 \mathrm{MG}^{\text {EGFRvIll }}$ cells that were intraperitoneally injected 4 hours before. Two hours 
after CAR-iMACs injection, whole animal imaging was conducted to capture the signal of DiR of the immune cells and bioluminescence from tumor cells. The following imagings were performed on day 1 , day 3 and day 7. PBS treated tumor-bearing mice were used as the control experiment. b, Exhibition of bioluminescence signals of the tumor-bearing mice after being treated by the three types of CAR-iMACs at different time points. $\mathbf{c}$, Statistics of the total fluorescence signal of each experimental group from $\mathbf{b}$, showing more strengthened anti-tumor activity of TIR-CAR-iMACs than the truncated CAR-iMACs and CD3ろ-CAR-iMACs. The data was shown by Graphpad Prism 8.2.1. $n \geq 5$. Error bar: Mean, SEM, N. Significance was calculated with two-way ANOVA analysis. p-value: 0.1234 (ns), $0.0332\left({ }^{*}\right), 0.0021(* *)$. d, The survival curve of CAR-iMACs-treated tumor-bearing mice from $b$, indicating that TIR-CAR-iMACs significantly improved the survival time of the treated mice. Recording and comparing the survival time of tumor-bearing mice after treatment using Graphpad Prism 8.2.1. $\mathrm{n} \geq 5$. Significance was calculated with Survival curve analysis. p-value: $<0.05(*),<0.01(* *),<0.001(\star \star *)$. 

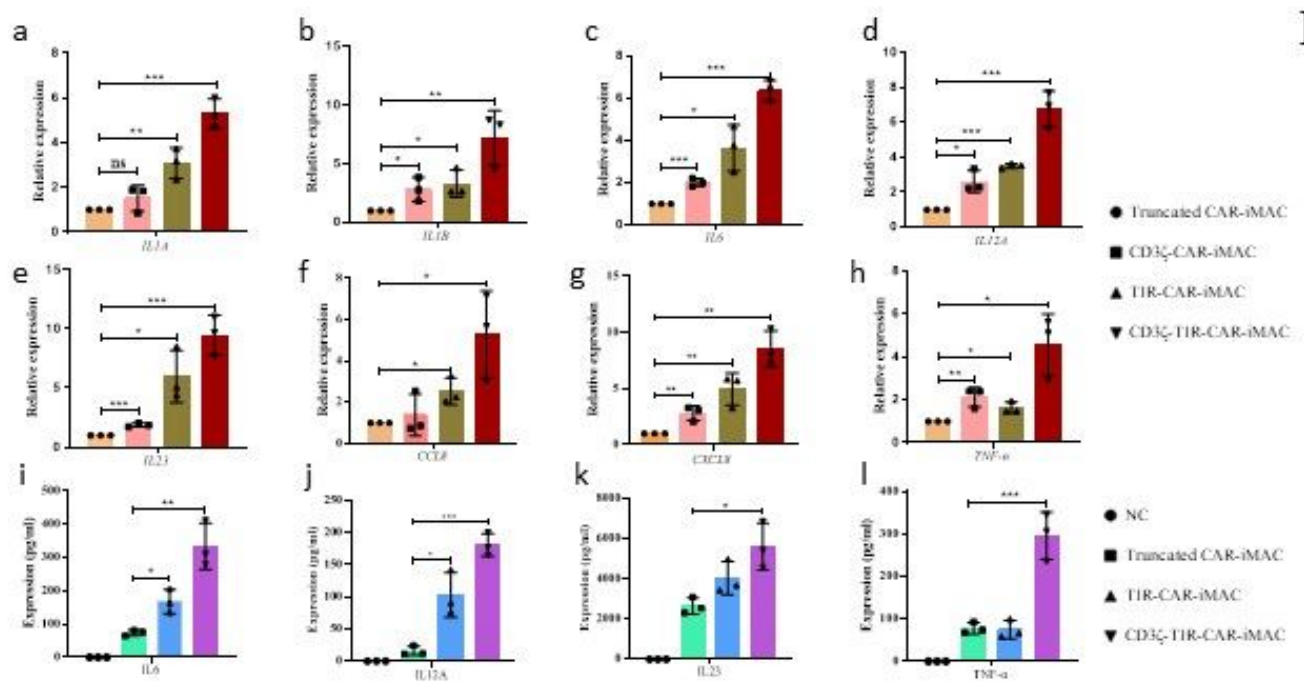

Figure 4
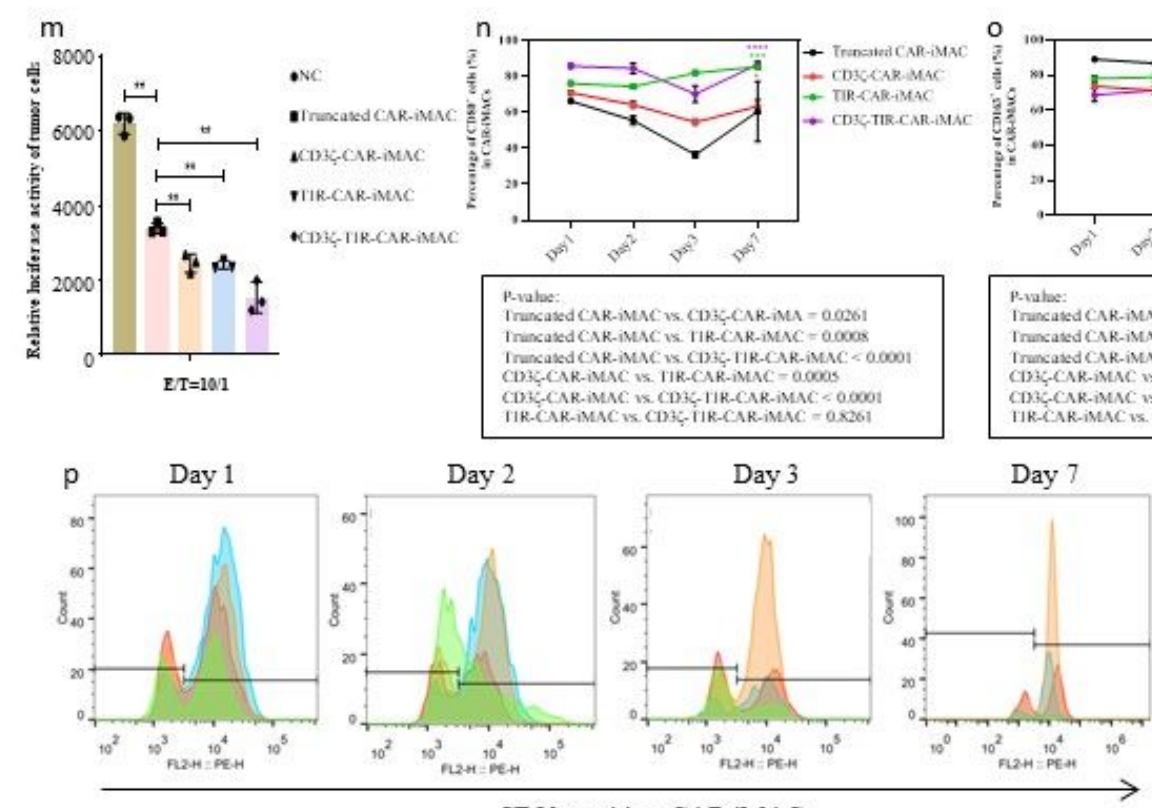

CD80-positive CAR-iMACs
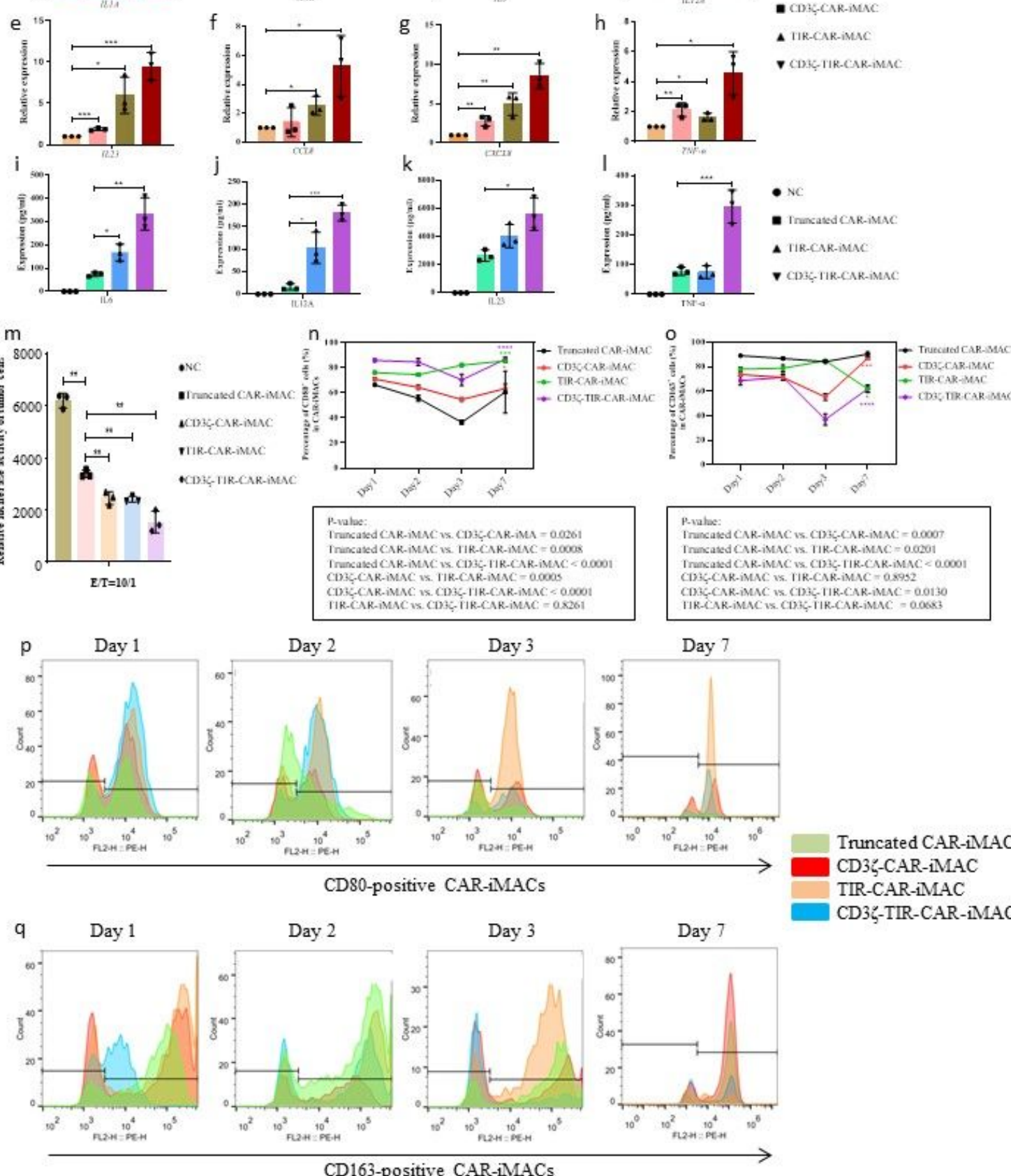

CD163-positive CAR-iMACs

\section{Figure 4}

Integration of TIR and CD3Z enhanced antigen-dependent immune activation, M1 polarization and cytotoxic effect of CAR-iMACs in vitro. a-h, qPCR analysis showing the expression of immune-related factor genes in CAR-iMACs. After co-culturing with U87MGEGFRvll cells for 24 hours with an E/T ratio of

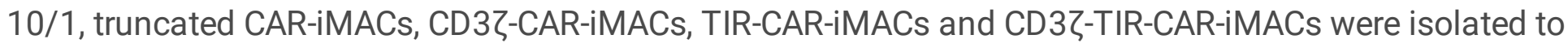
determine RNA expression of IL-1A (a), IL1B (b), IL6 (c), IL12A (d), IL23 (e), CCL8 (f), CXCL8 (g) and TNF-a 
(h). i-l, ELISA experiments showing the release of pro-inflammatory cytokines from CAR-iMACs in the medium. After 24 hours incubating of WT-iMACs, TIR-CAR-iMACs and CD3Z-TIR-CAR-iMACs with U87MG ${ }^{E G F R v I l l}$ cells respectively under the condition of an E/T ratio of $10 / 1$, the culture medium was harvested from the co-culture system and was utilized to detect IL6 (i), IL12A (j), IL23 (k) and TNF-a (I). The medium for the culturing of tumor cells without CAR-iMACs was used as a negative control (NC). $\mathrm{m}$, Statistics of bioluminescence from residual FFluc ${ }^{+}$U87MG ${ }^{\text {EGFRvIll }}$ cells after incubating with truncated

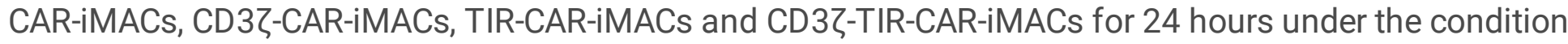
of an E/T ratio of 10/1. The experimental group containing the tumor cells without CAR-iMACs was used as a negative control (NC). All the data above was shown by Graphpad Prism 8.2.1. $n=3$. Error bar: Mean, SEM, N. Significance was calculated with Multiple t-tests analysis. p-value: 0.1234 (ns), $0.0332\left(^{*}\right)$,

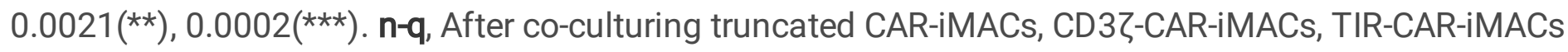
and CD3Z-TIR-CAR-iMACs with U87MG ${ }^{\text {EGFRvIll }}$ cells with an E/T ratio of 10/1, flow cytometry analyses were performed to detect the proportion of CD80- or CD163-positive CAR-iMACs at the time points of day 1 , day 2, day 3 and day 7 respectively. Statistic analyses showed the trends of the percentage of CD80positive CAR-iMACs ( $\mathbf{n}$ and $\mathbf{p}$ ) or CD163-positive CAR-iMACs ( $\mathbf{o}$ and $\mathbf{q}$ ) over the course of 7 days. .. The data was shown by Graphpad Prism 8.2.1. $n=3$. Error bar: Mean, SEM, N. Significance was calculated with two-way ANOVA analysis. p-value: 0.1234 (ns), 0.0332(*), 0.0021(**), $0.0002(* \star *), 0.0001(\star \star \star \star)$. 
Figure 5

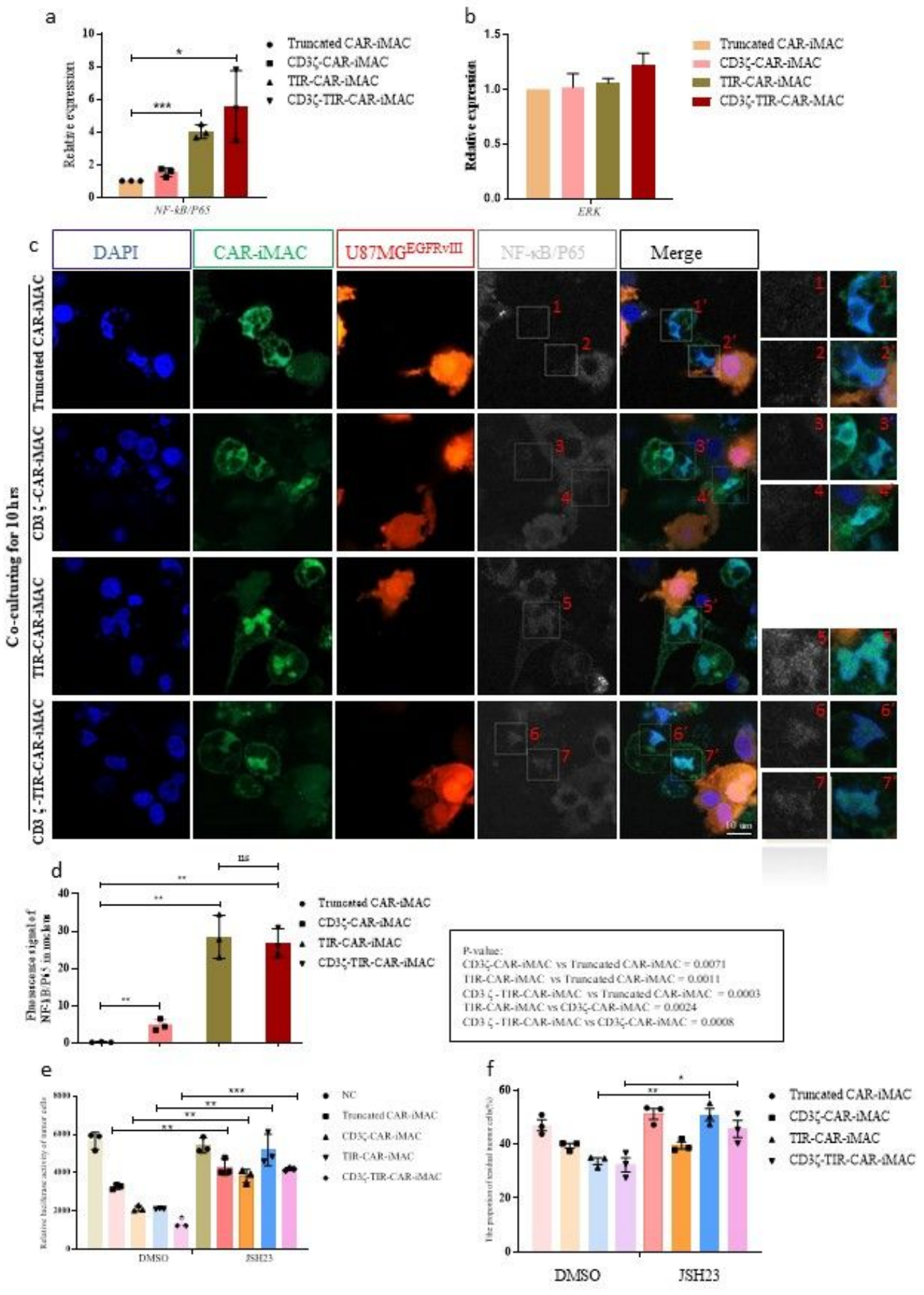

\section{Figure 5}

NF-KB/P65 mediated immune activation initiated by TIR domain-containing CARs. a and b, qPCR analysis showing the expression level of NF-KB/P65 encoding gene RELA(a) and ERK gene MAPK1 (b) in truncated CAR-iMACs, CD3Z-CAR-iMACs, TIR-CAR-iMACs and CD3Z-TIR-CAR-iMACs, after incubating the four different types of CAR-iMACs with U87MGEGFRvll cells for 10 hours without IFN-y/LPS pretreatment. c, Immunofluorescence staining indicating expression of NF-KB/P65 protein in nuclei of the four CAR- 
iMACs which were incubated with U87MG EGFRvIll cells for 10 hours without IFN-y/LPS pretreatment. $\mathbf{d}$, Statistical analysis of expression level of NF-KB/P65 protein in nuclei of the four CAR-iMACs as in C. The expression level of NF-KB/P65 protein in nuclei was detected with ImageJ software. $\mathbf{e}$, The residual luciferase-transduced tumor cells determined by luciferase activity after 24 hours of co-culturing with CAR-iMACs (without IFN- $y$ /LPS pretreatment) in the presence of JSH23 or DMSO. $\mathbf{f}$, The residual tdTotamo-transduced tumor cells determined by FCM, after 24 hours of co-culturing with CAR-iMACs (without IFN-y/LPS pretreatment) in the presence of JSH23 or DMSO. All the data was shown by Graphpad Prism 8.2.1. $n=3$. Error bar: Mean, SEM, N. Significance was calculated with multiple t-tests

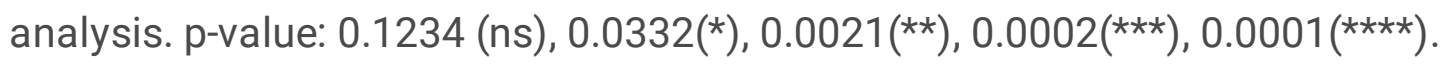




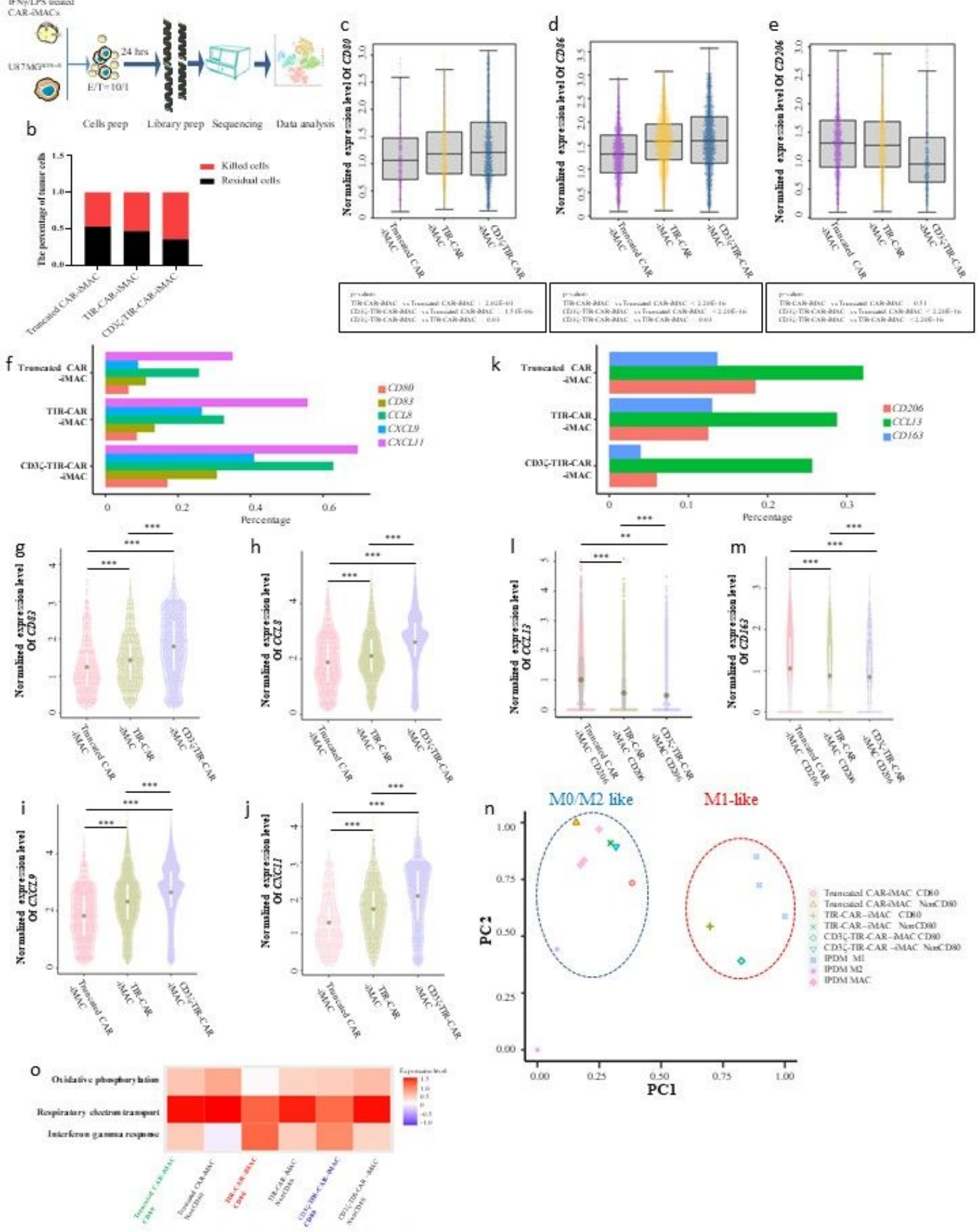

Figure 6

Single cell RNA-seq analysis revealed that TIR-based CARs promoted M1 polarization of CAR-iMACs. a, A schematic picture showing the procedures of the single cell RNA-seq experiment. $\mathbf{b}$, Statistics of percentage of residual U87MG ${ }^{\text {EGFRvill }}$ cells and eliminated that cells after co-culturing the cells with truncated CAR-iMACs, TIR-CAR-iMACs and CD3Z-TIR-CAR-iMACs respectively for 24 hours. ce, Statistics of expression levels of CD80 (C), CD86 (D), and CD163 (E) in truncated CAR-iMACs, TIR-CAR-iMACs and 
CD3Z-TIR-CAR-iMACs respectively. $\mathbf{f}$, Statistics of the percentage of three types of CAR-iMACs expressing M1 state-associated genes. The CD80 gene here was utilized as a positive control. g-j, Statistics of expression levels of M1 state-associated genes in the three types CAR-iMACs. $\mathbf{k}$, Statistics of the percentage of the three types CAR-iMACs expressing M2 state-associated genes. Similarly, the CD206 gene here was utilized as a positive control. I and $\mathbf{m}$, Statistics of cells numbers of the three types of CARiMACs expressing M2 state-associated genes. $\mathbf{n}$, Principle component analysis (PCA) showing the relationship between CAR-iMACs, and stimulated M1- and M2- iPSC derived macrophages (IPDM) ${ }^{31}$. Tomore accurately distinguish polarized and unpolarized CAR-iMACs, we further divided CAR-iMACs into CD80-positive and absent (NonCD80) categories. o, Heat map comparing activation levels of M1- and M2- related pathways in CD80-positive and absent (NonCD80) populations of the three types of CARiMACs. Oxidative phosphorylation and Respiratory electron transport activity were linked to the M2 state of macrophages. Interferon-gamma response was related to the M1 state. All the p-values are calculated by Mann-Whitney U test for unpaired samples (cells). p-value: $>0.05(n s),<0.05(*),<0.01(* *),<0.001(\star \star *)$.

\section{Supplementary Files}

This is a list of supplementary files associated with this preprint. Click to download.

- ReportingSummary.pdf

- Supplementaltable.docx 